\title{
Formulation of solid-shell finite elements with large displacements considering different transverse shear strains approximations
}

\author{
Fernando G. Flores ${ }^{a}$, Liz G. Nallim ${ }^{b}$, Sergio Oller ${ }^{c, d}$ \\ ${ }^{a}$ IDIT-Departamento de Estructuras, Universidad Nacional de Córdoba and CONICET, \\ Av. Velez Sarsfield 1611, 5016 Córdoba-Argentina, fernando.flores@unc.edu.ar, \\ te: 54-351-5353800 int 721 \\ ${ }^{b}$ Facultad de Ingenieria, INIQUI (CONICET), Universidad Nacional de Salta, Av. \\ Bolivia 5150, 4400 Salta, Argentina \\ ${ }^{c}$ CIMNE International Center for Numerical Method Engineering, Spain \\ ${ }^{d}$ UPC, Technical University of Catalonia (Barcelona Tech), Edif. C1, Campus Nord, \\ Jordi Girona 1-3, 08034 Barcelona, Spain
}

\begin{abstract}
This work presents a general formulation and implementation in solid-shell elements of the refined zigzag theory and the trigonometric shear deformation theory in an unified way. The model thus conceived is aimed for use in the analysis, design and verification of structures made of composite materials, in which shear strains have a significant prevalence. The refined zigzag theory can deal with composite laminates economically, adding only two nodal degrees of freedom, with very good accuracy. It assumes that the in-plane displacements have a piece-wise linear shape across the thickness depending on the shear stiffness of each composite layer. The trigonometric theory assumes a cosine variation of the transverse shear strain. A modification of this theory is presented in this paper allowing its implementation with $C^{0}$ approximation functions. Two existing elements are considered, an eightnode tri-linear hexahedron and a six-node triangular prism. Both elements use a modified right Cauchy-Green deformation tensor $\overline{\mathbf{C}}$ where five of its six components are linearly interpolated from values computed at the top and bottom surfaces of the element. The sixth component is computed at the center of the element and it is enhanced with an additional degree of freedom. This basic kinematic is improved with a hierarchical field of inplane displacement expressed in convective coordinates. The objective of this approach is to have a simple and efficient finite element formulation to analyze composite laminates under large displacements and rotations but
\end{abstract}


small elastic strains. The assumed natural strain technique is used to prevent transverse shear locking. An analytic through-the-thickness integration and one point integration on the shell plane is used requiring hourglass stabilization for the hexahedral element. Several examples are considered on the one hand to compare with analytical static solutions of plates, and on the other hand to observe natural frequencies, buckling loads and the non-linear large displacement behavior in double curved shells. The results obtained are in a very good agreement with the targets used.

Keywords: Solid-shell, Transverse shear, Composite laminate, Large displacements.

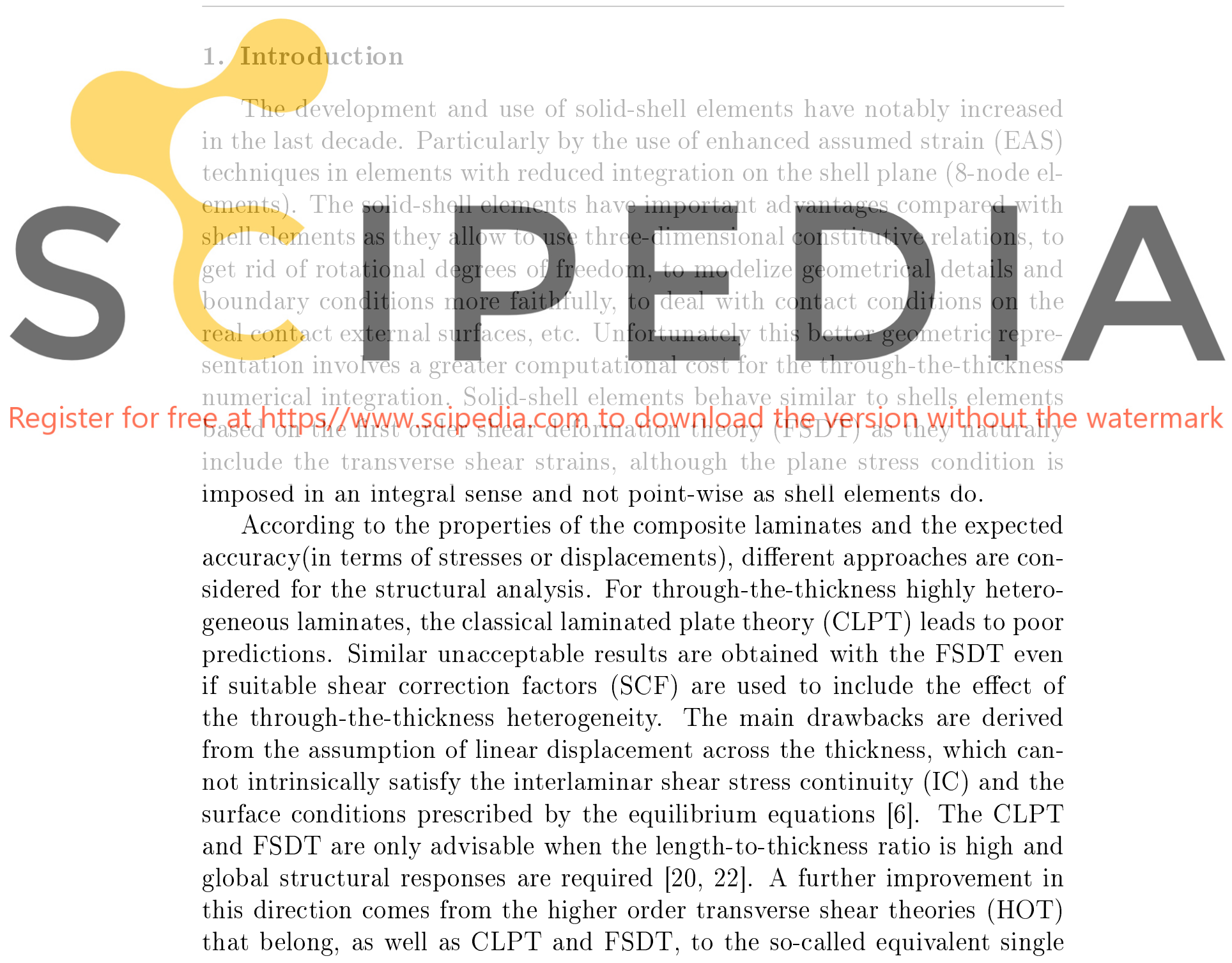


layer theories (ESL). In these theories, the in-plane displacements are suitable smooth functions of the transverse coordinate, with the number of the published shear shape functions assumption being large and varied (see for instance $[5,16,17,19,26,31,32])$. HOT are more accurate than CLPT and FSDT, but the continuity of the shear strains at interfaces leads to a discontinuity in the shear stress distribution, and although they do not require in principle the use of SCF, for highly heterogeneous laminates they lead to a very stiff behavior.

The most suitable technique for the analysis of composite materials is the use of three-dimensional solid finite elements. However it becomes prohibitively expensive as the number of layers in the laminate increase (it can be as large as one hundred), in optimization analysis or for non-linear problems. It is feasible to group multiple layers within one single layer with combined properties in order to maintain the number of degrees of freedom (DOFs) of the problem within manageable limits as suggested in [18]. The accuracy in transverse shear stresses can also be improved using hybrid elements including stresses as additional DOFs [35].
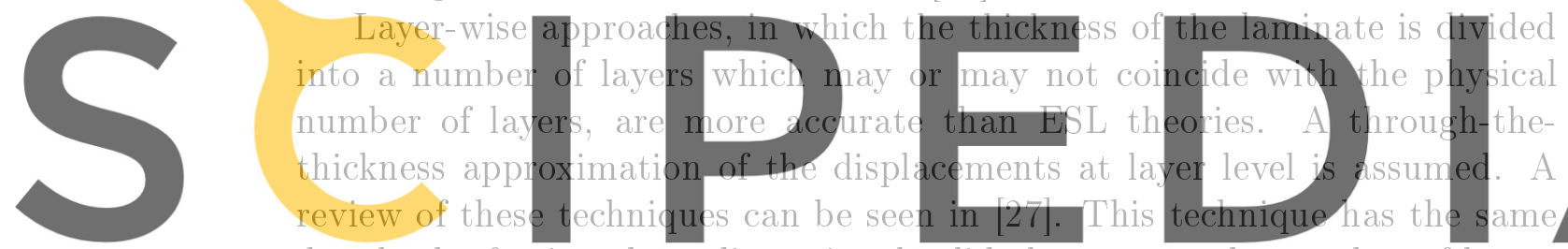

drawback of using three dimensional solid elements as the number of layers

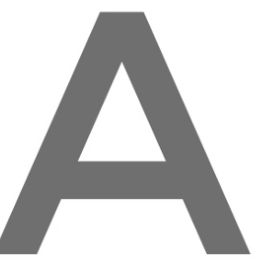

Register for free at https//www.scipedia.com to download the version without the watermark For angle-ply laminates and those with a low order of heterogeneity one

can consider a smooth transverse shear variation across the thickness as pro-

posed by HOT including, for instance, the trigonometric shear deformation theory (TSDT) $[5,17,24,25]$.

For sections with a high degree of heterogeneity, the analysis with solid models and layer-wise approaches shows that the in-plane displacements profiles are far from a smooth curve that could be approximated by a polynomial of third order or higher. This has led to the so called zigzag theories where the in-plane displacements functions are only $C^{o}$ continuous with a zigzag profile, possibly with strong discontinuities in the derivatives (associated to the transverse shear strain) to fit the IC of adjacent layers with shear modulus that can differ by several orders of magnitude. A review of the evolution of these theories can be seen in [2]. More recently, a refined version of this approach has been presented [28], where two hierarchical DOFs are added to the five DOFs of the FSDT enhancing the linear through-the-thickness interpolation. This approach leads to constant transverse shear stresses at each layer (i.e. discontinuous) as they are computed from the constitutive 
equations; however it allows dealing with clamped boundary conditions, a limitation of the previous zigzag theories in which it is based on.

This refined zigzag theory (RZT), that makes the use of SCF unnecessary, has been implemented in 2D beam finite elements [12, 23, 3, 21], in flat plate finite elements $[28,4,13,34,1]$ where a very good approximation to the in-plane displacements has been reported, in shell finite elements with linear kinematics [33] and with large displacements and small strains [8]. The piece-wise constant transverse shear stresses calculated directly from the computed strains and the constitutive relations for each layer show frequently a poor approximation. An accurate evaluation of shear stresses requires the through-the-thickness integration of the in-plane equilibrium equations, which involves ad hoc schemes for the computation of the derivatives of the in-plane stresses between finite elements. To avoid the a posteriori integration of the equilibrium conditions and to improve the predicting capabilities of the RZT, a mixed approach has been developed for beams with linear kinematics[30]. Based on the latter a so denoted RZT (3,2) $_{\text {mas }}$ been developed for beams[15], where the mixed approach is combined with im-
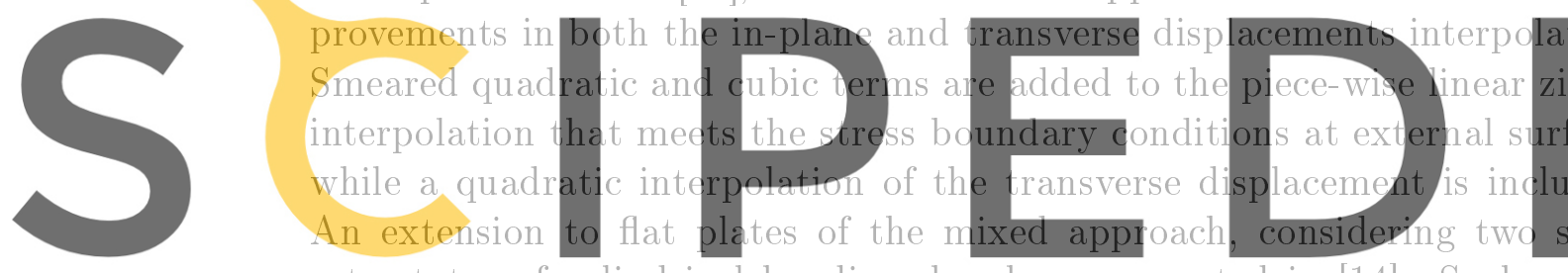

rate states of cylindrical bending, has been presented in [14]. Such mixed

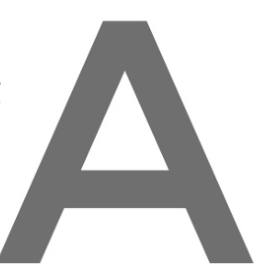

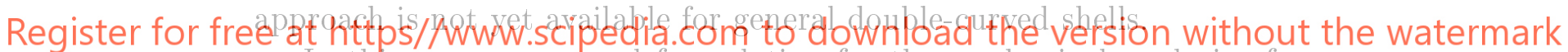

In this paper a general formulation for the mechanical analysis of com-

posite laminated structures is proposed. The model employs solid-shell finite

elements with large displacements and considers different transverse shear strains approximations in a unified way. The elements considered are a trilinear 8-node hexahedron[9] and a 6-node triangular prism[7] in which two refined zigzag approaches (RZT), and a HOT (TSDT) are implemented. To attain this purpose the TSDT kinematic is modified and suited to work with FSDT-shell and solid-shell finite elements. In addition, this modification allows to unify the general formulation presented in this paper. The scope of this work is restricted to small elastic strains but large displacements and rotations.

An outline of this paper is as follows. Next section provides a short description of the formulation of the solid-shell elements considered. Then the additional displacement fields and the associated strains are introduced. Resulting elasticity matrices for the new generalized stress and strain measures are then evaluated. Several examples are presented in Section 5 to show the 
very good correlation with theoretical results, with shell models and with 3D solid discretizations. Finally some conclusions are summarized.

\section{Solid-Shell Elements}

Two prismatic solid-shell elements are considered, namely a triangular $(N N=6)$ and a quadrilateral $(N N=8)$ based one. The original and deformed geometries of the element are described by the standard isoparametric approximations [36].

$$
\mathrm{x}(\xi)=\sum_{I=1}^{N N} N^{I}(\xi) \mathrm{x}^{I}=\sum_{I=1}^{N N} N^{I}(\xi)\left(\mathrm{X}^{I}+\mathrm{u}^{I}\right)
$$

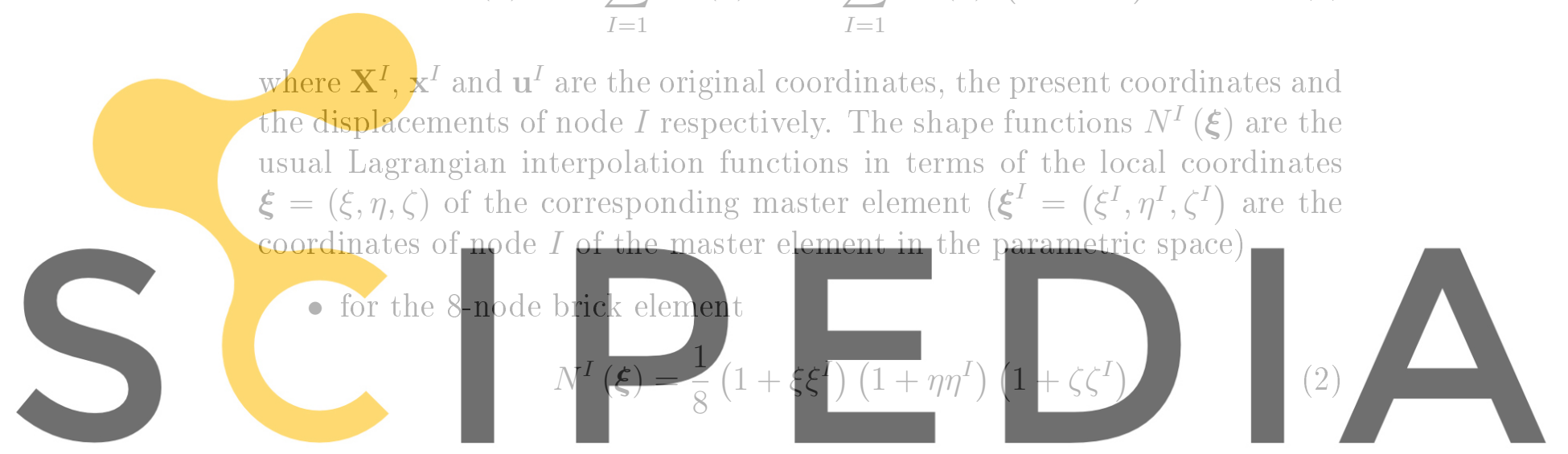

- for the 6 node prism element the in-plane interpolation uses area coor-

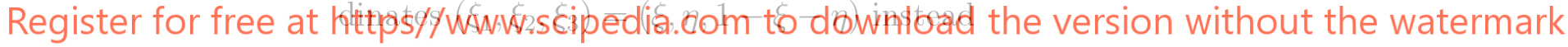

$$
\begin{aligned}
& N^{I}(\xi)=\frac{1}{2}(1-\zeta) \xi_{I} \quad I=1 . .3 \\
& N^{I}(\boldsymbol{\xi})=\frac{1}{2}(1+\zeta) \xi_{I-3} \quad I=4 . .6
\end{aligned}
$$

Following a standard approach, at each point of interest the Cartesian derivatives are computed using the Jacobian matrix

$$
\begin{aligned}
& \mathbf{J}=\frac{\partial \mathbf{X}}{\partial \boldsymbol{\xi}}=\mathbf{X}_{, \boldsymbol{\xi}} \\
& N_{, \mathbf{X}}^{I}=\mathbf{J}^{-1} N_{, \boldsymbol{\xi}}^{I}
\end{aligned}
$$

At each element center a local Cartesian system is defined with the first two axes in the plane tangent to the shell mid-surface, that may coincide with principal directions of the laminate or any other convenient condition (see Figure 2)

$$
\mathbf{R}=\left[\mathbf{t}_{1}, \mathbf{t}_{2}, \mathbf{t}_{3}\right]
$$



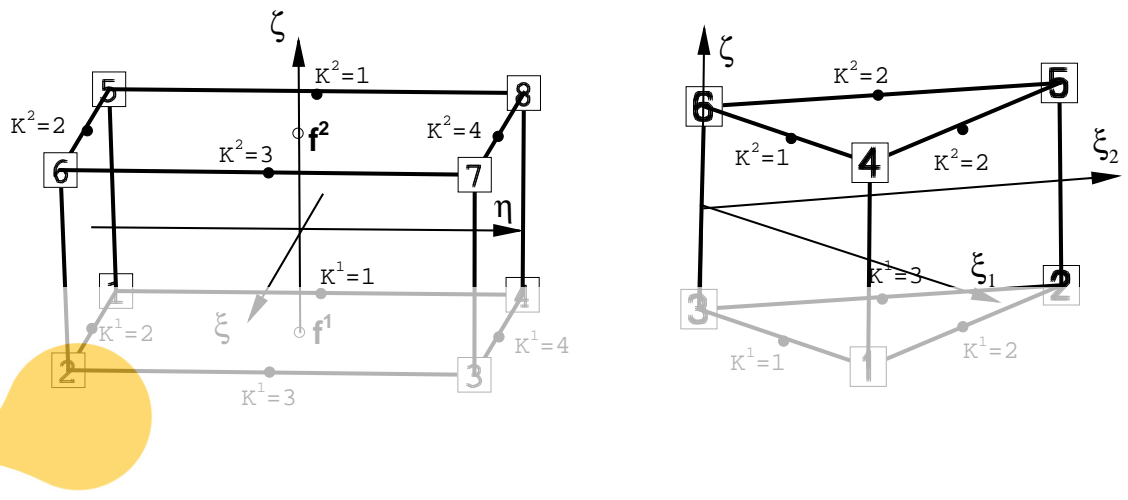

Figure 1: Solid-Shell Elements. Parametric space and Sampling points
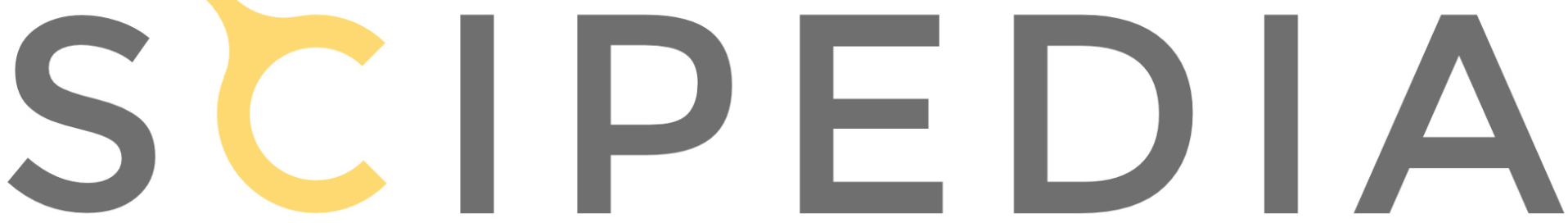

Register for free at https//www.scipedia.com to download the version without the watermark
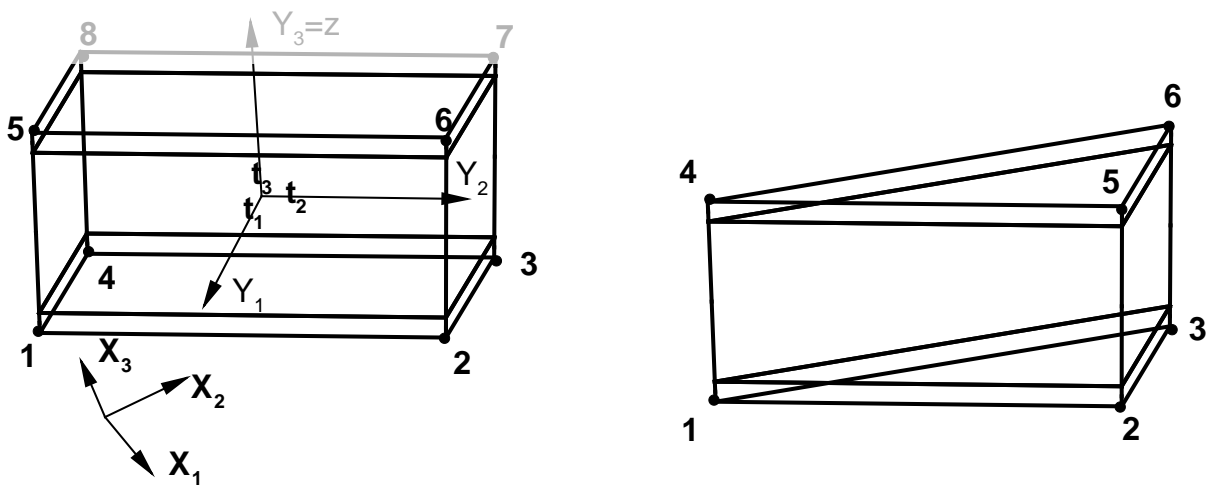

Figure 2: Solid-Shell Elements. Node numeration and layer orientation. 
such that Cartesian derivatives with respect to this local system $(\mathbf{Y})$ are

$$
N_{, \mathbf{Y}}^{I}=\mathbf{R}^{T} N_{, \mathbf{X}}^{I}
$$

that allows to compute the deformation gradient $\mathbf{F}$ in terms of the present nodal coordinates (Latin indices $i, j=1 . .3$ )

$$
F_{i j}=\sum_{I=1}^{N N} N_{, Y_{j}}^{I} x_{i}^{I}
$$

and the components of the right Cauchy-Green tensor $\mathbf{C}$

$$
C_{i j}=F_{k i} F_{k j}
$$

from which the Green-Lagrange strain tensor can be computed as

$$
E_{i j}=\frac{1}{2}\left(C_{i j}-\delta_{i j}\right)
$$
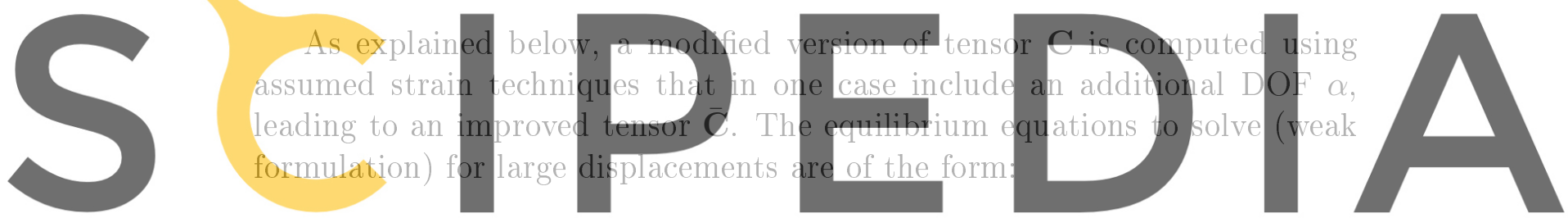

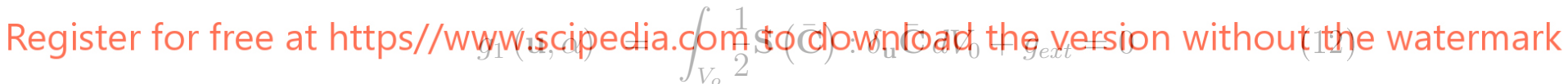

$$
g_{2}(\mathbf{u}, \alpha)=\int_{V_{o}} \frac{1}{2} \mathbf{S}(\overline{\mathbf{C}}): \delta_{\alpha} \overline{\mathbf{C}} d V_{0}=0
$$

where $\mathbf{S}$ is the second Piola-Kirchhoff stress tensor. The second equation is solved at element level and allows to condense the additional DOF $\alpha$ in the solution scheme.

\subsection{Improvements of the standard elements}

To use these solid elements in large-displacements shell problems their formulation must be substantially improved. In the sequel a modified right Cauchy-Green $\overline{\mathbf{C}}$ tensor is introduced where its components, as well as the components of the deformation gradient, are all written in the local system defined in (7).

The discretization process of a laminate with solid elements involves two steps: a) a discretization of the shell middle surface with triangles or quadrilaterals, b) a discretization in the thickness direction with one or more solid 
elements from the element defined on the middle surface. It will be assumed that the element connectivities are introduced with the first local nodes 1 to $N F(=N N / 2)$ associated with the lower surface and the last $N F$ nodes are above the first ones in the thickness direction at a distance equal to the laminate thickness or a fraction of it in case more than one element are used. In this way the surface normal direction is practically coincident with the third component $\boldsymbol{t}_{3}$ of local system defined in (7) (see Figures 1-2).

As the strain measure considered above for the solid element is defined in term of the right Cauchy-Green tensor, an interesting possibility is to modify the components of $\mathbf{C}$ (or $\mathbf{E}$ ) directly associated to the behavior to be improved. Distinguishing each component of $\mathbf{C}$ with a superscript

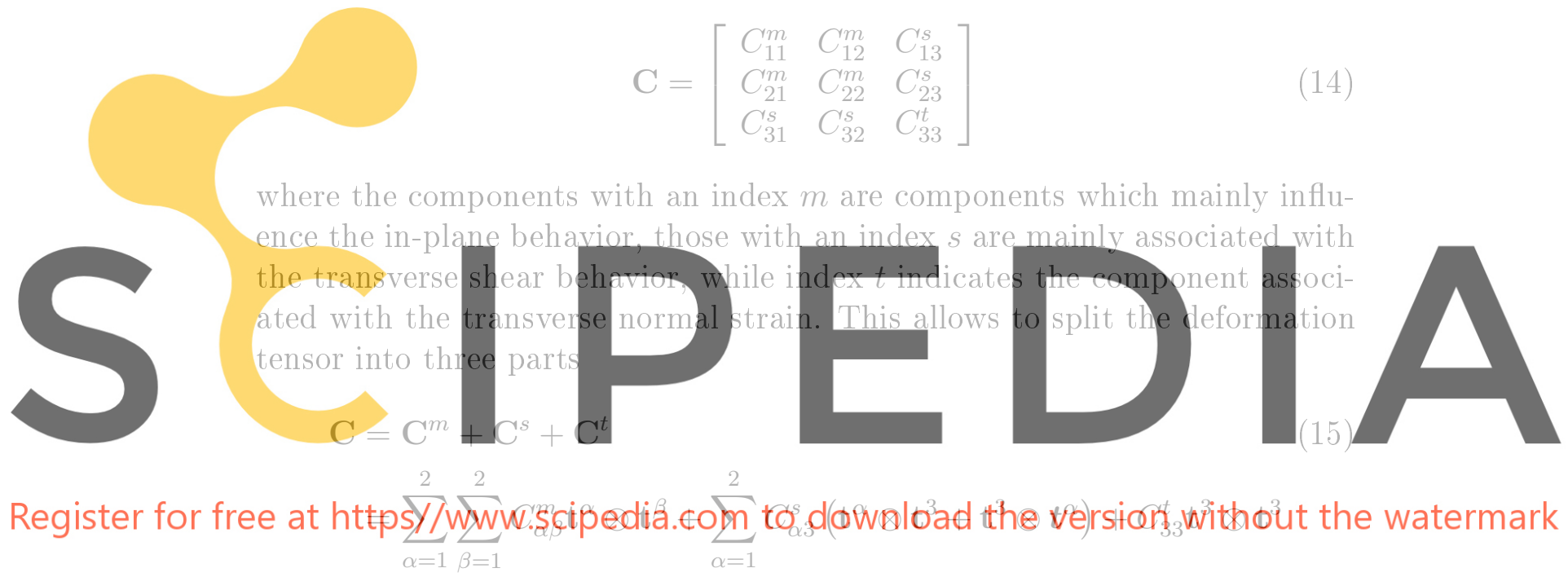

\subsection{In-plane behavior}

The membrane and bending behavior are defined in terms of the in-plane components of $\mathbf{C}$ computed at the center of the lower and upper surfaces. The values at the center of each face $f$ are calculated as the weighted average of the values computed at each mid-side point of the face (Greek indices $\alpha, \beta=1 . .2)$ :

$$
\bar{C}_{a \beta}^{f}=\frac{1}{A^{f}} \sum_{K=1}^{N F} J_{K} C_{\alpha \beta}^{K} \quad \sum_{K=1}^{N F} J_{K}=A^{f}
$$

where $J_{K}$ is the Jacobian determinant at each mid-side point $K$. Figure 1 shows the sampling points $K^{1}=1 . . N F$ at the bottom face $\left(f^{1}\right)$ and sampling points $K^{2}=1 . . N F$ for the upper face $\left(f^{2}\right)$ for both element types. This average approximation of the first fundamental form of each face surface 
may be seen as an assumed strain approach. The in-plane components of the Green-Lagrange strain tensor at the face center can be written as

$$
\begin{aligned}
\bar{E}_{\alpha \beta} & =\frac{1}{2} \frac{1}{A^{f}} \sum_{K=1}^{N F} J_{K}\left[C_{\alpha \beta}^{K}-{ }^{o} C_{\alpha \beta}^{K}\right] \\
& =\frac{1}{2}\left(\bar{C}_{\alpha \beta}-\delta_{\alpha \beta}\right)
\end{aligned}
$$

with $\delta_{\alpha \beta}$ the Kronecker delta and the left index " $O$ " denotes that is computed at the original configuration. The in-plane components of the right CauchyGreen tensor at each face are linearly interpolated across the thickness:

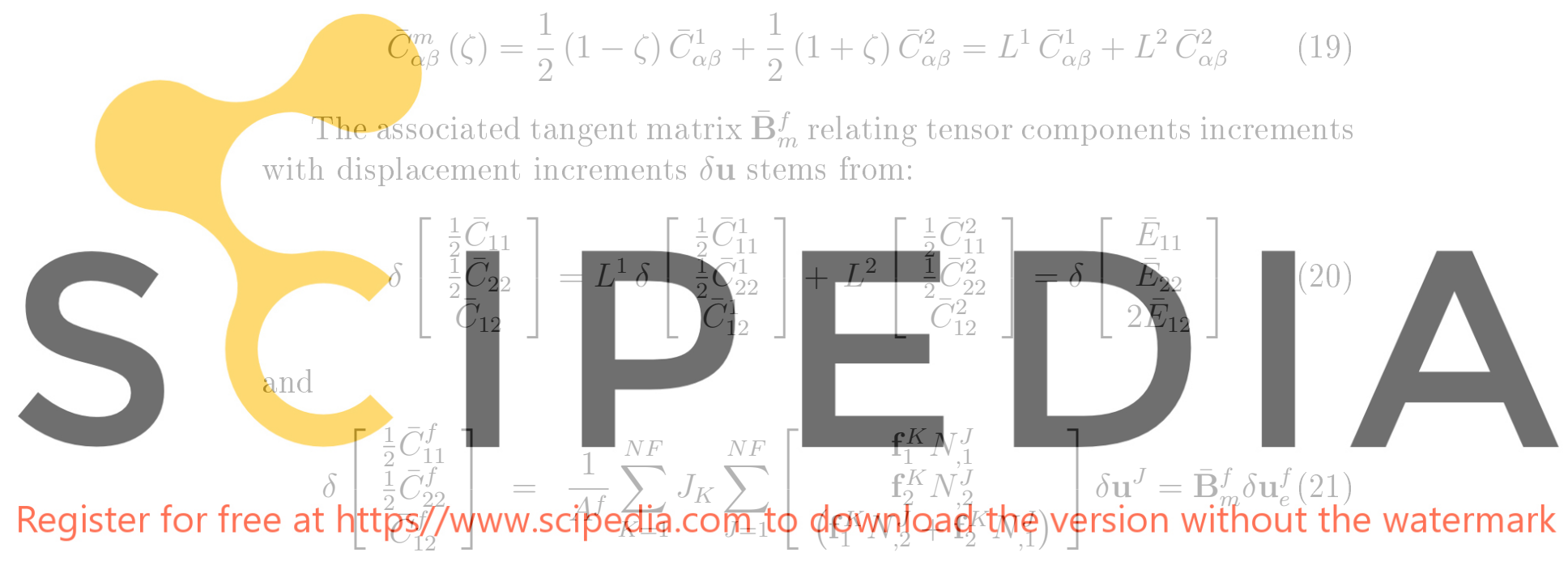

where the vector $\delta \mathbf{u}_{e}^{f}$ includes the nodes of the face $f$ (lower or upper) only. Then we can write as follows

$$
\left[\overline{\mathbf{B}}_{m}\right]_{3 \times(3 N N)}=\left[\begin{array}{ll}
L^{1} \overline{\mathbf{B}}_{m}^{1} & L^{2} \overline{\mathbf{B}}_{m}^{2}
\end{array}\right]
$$

Note that each matrix is associated with a different set of nodes, as matrix $\overline{\mathbf{B}}_{m}^{1}\left(\overline{\mathbf{B}}_{m}^{2}\right)$ multiplies only DOFs of the nodes in the lower (upper) face.

Furthermore, assuming a constant Jacobian determinant across the thickness (that strictly requires the same discretization of both lower and upper surface and a constant thickness), and comparing with the strain measures of shell theories, it is possible to compute the Green-Lagrange strain tensor at the middle surface and the changes of curvatures as

$$
\begin{aligned}
\mathbf{E}_{m} & =\frac{1}{2}\left(\overline{\mathbf{E}}_{f}^{1}+\overline{\mathbf{E}}_{f}^{2}\right) \\
\boldsymbol{\chi}_{b} & =\frac{1}{h}\left(\overline{\mathbf{E}}_{f}^{2}-\overline{\mathbf{E}}_{f}^{1}\right)
\end{aligned}
$$


that allows to write the in-plane strains across the thickness as (with $z \in$ $\left[-\frac{h}{2},+\frac{h}{2}\right]$ the local Cartesian coordinate in the normal direction):

$$
\overline{\mathbf{E}}(z)=\mathbf{E}_{m}+z \chi_{b}
$$

while the associated tangent matrices can respectively be written as (keep in mind that each $\overline{\mathbf{B}}_{m}^{f}$ is associated to a different set of DOFs):

$$
\begin{aligned}
\overline{\mathbf{B}}_{m} & =\frac{1}{2}\left[\overline{\mathbf{B}}_{m}^{1}, \overline{\mathbf{B}}_{m}^{2}\right] \\
\overline{\mathbf{B}}_{b} & =\frac{1}{h}\left[-\overline{\mathbf{B}}_{m}^{1}, \overline{\mathbf{B}}_{m}^{2}\right]
\end{aligned}
$$

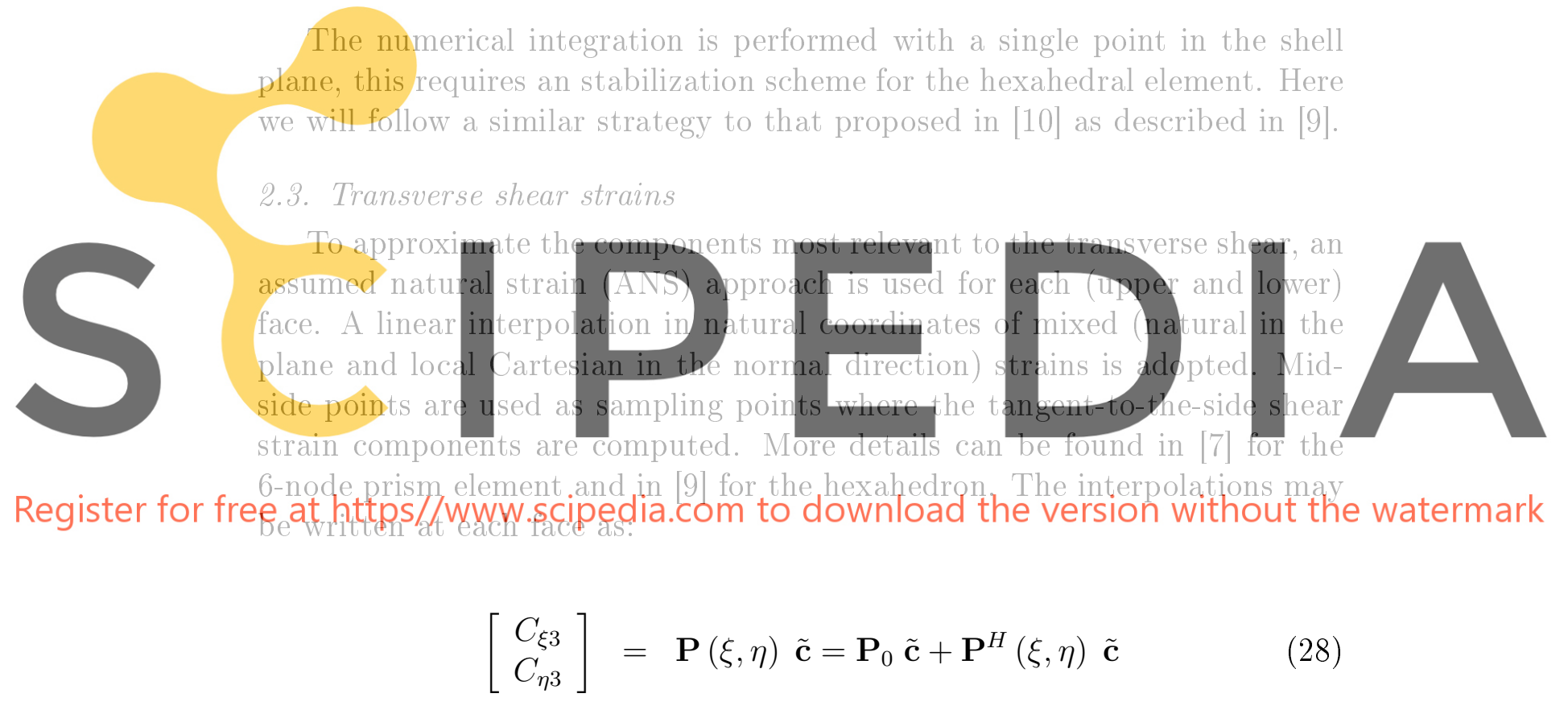

where $\tilde{\boldsymbol{c}}$ gathers the shear strains at the sampling points and $\mathbf{P}$ are the linear (in terms of natural coordinates) interpolation polynomials. These polynomials $\mathbf{P}$ are evaluated at the center of each face for the numerical integration $\left(\boldsymbol{P}_{0}\right)$ and the resulting strains are linearly interpolated across the thickness. The difference $\left(\mathbf{P}-\mathbf{P}_{0}=\mathbf{P}^{H}\right)$ is used for hourglass stabilization purposes in the case of the hexahedron. The interpolated components in (28) allows to write

$$
\overline{\mathbf{C}}^{s}=C_{\xi 3}\left(\mathbf{t}^{\xi} \otimes \mathbf{t}^{3}+\mathbf{t}^{3} \otimes \mathbf{t}^{\xi}\right)+C_{\eta 3}\left(\mathbf{t}^{\eta} \otimes \mathbf{t}^{3}+\mathbf{t}^{3} \otimes \mathbf{t}^{\eta}\right)
$$

where $\left[\begin{array}{lll}\mathbf{t}^{\xi} & \mathbf{t}^{\eta} & \mathbf{t}^{3}\end{array}\right]$ are the dual base vectors of the local mixed triad $\left[\begin{array}{lll}\mathbf{t}_{\xi} & \mathbf{t}_{\eta} & \mathbf{t}_{3}\end{array}\right]=\left[\frac{\partial \mathbf{X}}{\partial \xi} \frac{\partial \mathbf{X}}{\partial \eta} \frac{\partial \mathbf{X}}{\partial y_{3}}\right]$ that allows to compute the modified (improved) 
Cartesian components

$\bar{C}_{\alpha 3}=\mathbf{t}_{\alpha} \cdot \overline{\mathbf{C}}^{s} \cdot \mathbf{t}_{3}=\mathbf{t}_{\alpha} \cdot\left[C_{\xi 3}\left(\mathbf{t}^{\xi} \otimes \mathbf{t}^{3}+\mathbf{t}^{3} \otimes \mathbf{t}^{\xi}\right)+C_{\eta 3}\left(\mathbf{t}^{\eta} \otimes \mathbf{t}^{3}+\mathbf{t}^{3} \otimes \mathbf{t}^{\eta}\right)\right] \cdot \mathbf{t}_{3}$

where denoting by $a_{i}^{j}=\mathbf{t}_{i} \cdot \mathbf{t}^{j}$ (with $i=1,2,3$ and $j=\xi, \eta, 3$ ) we can write

$$
\bar{C}_{\alpha 3}=C_{\xi 3}\left(a_{\alpha}^{\xi} a_{3}^{3}+a_{1}^{3} a_{3}^{\xi}\right)+C_{\eta 3}\left(a_{\alpha}^{\eta} a_{3}^{3}+a_{1}^{3} a_{\eta}^{3}\right)=C_{\xi 3} a_{\alpha}^{\xi}+C_{\eta 3} a_{\alpha}^{\eta}
$$

Finally using the condition $a_{i}^{3}=\delta_{i}^{3}$ we have

$$
\left[\begin{array}{l}
\bar{C}_{13} \\
\bar{C}_{23}
\end{array}\right]=\left[\begin{array}{ll}
a_{1}^{\xi} & a_{1}^{\eta} \\
a_{2}^{\xi} & a_{2}^{\eta}
\end{array}\right]\left[\begin{array}{l}
C_{\xi 3} \\
C_{\eta 3}
\end{array}\right]=\mathbf{J}_{p}^{-1}\left[\begin{array}{c}
C_{\xi 3} \\
C_{\eta 3}
\end{array}\right]
$$

where $\mathbf{J}_{p}^{-1}$ is the inverse of the Jacobian matrix of the isoparametric interpolation restricted to the tangent plane in each face. Then these values are interpolated across the thickness at the element axis.

$$
\overline{\mathbf{C}}^{s}=L^{1} \overline{\mathbf{C}}^{s 1}+L^{2} \overline{\mathbf{C}}^{s 2}
$$

where $\overline{\mathbf{C}}^{s f}$ results from (32) computed at face $f$.

\subsection{Transverse normal strain}

To avoid numerical locking due to the Poisson effect (transverse normal strain due to in-plane strains) an enhanced assumed strain (EAS) approach is considered. Note first that at the element center the component $C_{33}^{C}$ can be computed from

$$
\mathbf{f}_{3}^{C}=\sum_{I=1}^{N N} N_{, 3}^{I C} \mathbf{x}^{I}
$$

then the enhanced version is defined here as:

$$
\bar{C}_{33}=\mathbf{f}_{3}^{C} \cdot \mathbf{f}_{3}^{C}+2 \alpha z=C_{33}^{C}+2 \alpha z
$$

For the linear interpolation (1) the transverse component $C_{33}^{C}$ is constant in the normal direction. With the enhanced version

$$
\bar{E}_{33}(z)=\frac{1}{2}\left(\bar{C}_{33}-1\right)=E_{33}^{C}+\alpha z
$$

The variation of this Green-Lagrange strain component is

$$
\begin{aligned}
\delta \bar{E}_{33} & =\frac{1}{2} \delta \bar{C}_{33}=\delta \mathbf{f}_{3}^{C} \cdot \mathbf{f}_{3}^{C}+z \delta \alpha \\
& =\sum_{I=1}^{N N} N_{, 3}^{I} \mathbf{f}_{3}^{C}+z \delta \alpha=\mathbf{B}_{3}^{C} \delta \mathbf{u}_{e}+z \delta \alpha
\end{aligned}
$$


where $N_{, 3}^{I}=\frac{\partial N^{I}}{\partial Y_{3}}$ are the shape functions derivatives with respect to the shell normal coordinate computed at the element center, $\mathbf{f}_{3}^{C}=\left.\frac{\partial \mathbf{x}}{\partial Y_{3}}\right|_{\boldsymbol{\xi}=\mathbf{0}}$ is the deformation gradient component in the normal direction also computed at the element center and $\delta \mathbf{u}_{e}$ gathers the displacements of the $N N$ nodes of the element (i.e. $\left[\delta \mathbf{u}_{e}\right]^{T}=\left\{\left[\delta \mathbf{u}_{e}^{f 1}\right]^{T},\left[\delta \mathbf{u}_{e}^{f 2}\right]^{T}\right\}$ ).

Note that for a flat plate with linear kinematics, equation (36) can be seen as

$$
\begin{aligned}
\bar{E}_{33}(z) & =E_{33}^{0}+\alpha z=\frac{\partial u_{z}}{\partial z} \\
& =\frac{u_{z}^{T}-u_{z}^{B}}{h}+\alpha z=\frac{\partial u_{z}}{\partial z}
\end{aligned}
$$

where $u_{z}^{T}$ and $u_{z}^{B}$ are the normal displacement at the top and bottom surface respectively. This expression can be integrated across the thickness leading to

$$
\begin{aligned}
u_{z}(z) & =u_{z}^{B}+\int_{-h / 2}^{z}\left(\frac{u_{z}^{T}-u_{z}^{B}}{h}+\alpha z\right) d z \\
& =u_{z}^{B} \frac{1}{2}\left(1-\frac{2 z}{h}\right)+u_{z}^{T} \frac{1}{2}\left(1+\frac{2 z}{h}\right)-\frac{\alpha h^{2}}{8}\left[1-\left(\frac{2 z}{h}\right)^{2}\right]
\end{aligned}
$$

that allows to interpret the EAS parameter $\alpha$ as an element-wise (nonconforming) hierarchical DOF $\Delta u_{z}=-\frac{\alpha h^{2}}{8}$ at the element center.

\section{Additional displacement field}

The above described elements have a linear variation across the thickness of all the strain components. To enhance such interpolation, an additional (hierarchical) displacement vector field is included. These additional displacements are introduced in the local convective coordinate system with components in the tangent plane $\left(Y_{1}, Y_{2}\right)$ only, namely

$$
\begin{aligned}
{\left[\begin{array}{l}
u_{1}^{a}\left(Y_{1}, Y_{2}, Y_{3}\right) \\
u_{2}^{a}\left(Y_{1}, Y_{2}, Y_{3}\right)
\end{array}\right] } & =\sum_{I=1}^{N F} N^{I}\left(Y_{1}, Y_{2}\right)\left[\begin{array}{ll}
\phi_{1}\left(Y_{3}\right) & \\
& \phi_{2}\left(Y_{3}\right)
\end{array}\right]\left[\begin{array}{l}
\psi_{1}^{I} \\
\psi_{2}^{I}
\end{array}\right] \\
\mathbf{u}^{a}\left(Y_{1}, Y_{2}, Y_{3}\right) & =\sum_{I=1}^{N F} N^{I}\left(Y_{1}, Y_{2}\right) \boldsymbol{\phi}\left(Y_{3}\right) \boldsymbol{\psi}^{I}
\end{aligned}
$$

with the condition that the associated shape functions $\phi_{\alpha}(z)$ are null at both upper and lower surfaces

$$
\phi\left( \pm \frac{h}{2}\right)=0
$$


The functions $\phi_{\alpha}$ have units of length while the amplitude $\psi_{\alpha}$ is nondimensional. Three options has been considered here for $\phi$, two are associated with refined zigzag theories and the third one with the trigonometric shear deformation theory. The RZT splits the transverse shear stresses into a discontinuous and a continuous part

$$
\tau_{\alpha z}^{k}(z)=G_{\alpha z}^{k} \eta_{\alpha}+G_{\alpha z}^{k}\left[\left(1+\beta_{\alpha}^{k}\right) \psi_{1}\right]=G_{\alpha z}^{k} \eta_{\alpha}+\bar{\tau}_{\alpha z}^{k}(z)
$$

where $\eta_{\alpha}=\gamma_{\alpha}^{b}-\psi_{\alpha}$ is a shear strain measure (with $\gamma_{\alpha}^{b}$ the transverse shear strain computed from the base displacements (1) alone) constant or linear across the thickness (and thus leading to stress discontinuities between layers), while $\bar{\tau}_{\alpha z}^{k}(z)$ is assumed continuous. The first option, denoted here by RZT, is the approach in the original work or Tessler [28] where the slopes $\beta_{\alpha}=\phi_{\alpha, z}$ are assumed constant at each layer thus allowing to compute $\beta_{\alpha}^{k}$ as a function of the shear elasticity modulus $G_{\alpha z}$ for each direction $\mathbf{t}_{\alpha}$ of the composite laminate. Denoting by $\phi_{\alpha}^{k}=\phi_{\alpha}\left(z^{k}\right)$ (with $z^{k}$ the coordinate of the top of layer $k$, see Figure 3.a for an example), it can be found that:

$$
\begin{aligned}
\int_{h} \beta d z & =\phi^{N}-\phi^{0}=\sum \beta_{\alpha}^{k} h_{k}=0 \\
\beta_{\alpha}^{k} & =\frac{\phi_{\alpha}^{k}-\phi_{\alpha}^{k-1}}{h_{k}}=\frac{h}{G_{\alpha z}^{k} \sum \frac{h_{i}}{G_{\alpha z}^{i}}}-1 \\
\phi_{\alpha}(z) & =\phi_{\alpha}^{k-1}+\beta_{\alpha}^{k}\left(z-z^{k-1}\right) \quad z^{k-1} \leq z \leq z^{k}
\end{aligned}
$$

It has been shown that this piece-wise linear approximation leads to very good results for sandwich sections with large ratios between the $G_{\alpha z}^{k}$ of each layer.

The second RZT approach, that will be denoted here by $\mathrm{RZT}_{(3)}$, is due to Iurlaro et al [15]. In this case the continuous $\bar{\tau}_{\alpha z}^{k}(z)$ is additionally constrained to nullify at both external surfaces. These two additional conditions are satisfied by augmenting the piece-wise linear zigzag function with smeared quadratic and cubic terms (see [15] for details):

$$
\begin{aligned}
\phi_{\alpha}(z) & =\varphi_{\alpha}^{k}\left(z^{k-1}\right)+\rho_{\alpha}^{k}\left(z-z^{k-1}\right)+\chi_{0} z^{2}+\omega_{0} z^{3} \quad z^{k-1} \leq z \leq z^{k} \\
\rho_{\alpha}^{k} & =\frac{\varphi_{\alpha}^{k}-\varphi_{\alpha}^{k-1}}{h_{k}}
\end{aligned}
$$

The third option is a sinusoidal function that will be written as $\left(\phi_{1}=\phi_{2}\right.$ in this case):

$$
\begin{aligned}
& \phi(z)=h \sin \left(\frac{\pi z}{h}\right)-2 z \\
& \beta(z)=\pi \cos \left(\frac{\pi z}{h}\right)-2
\end{aligned}
$$




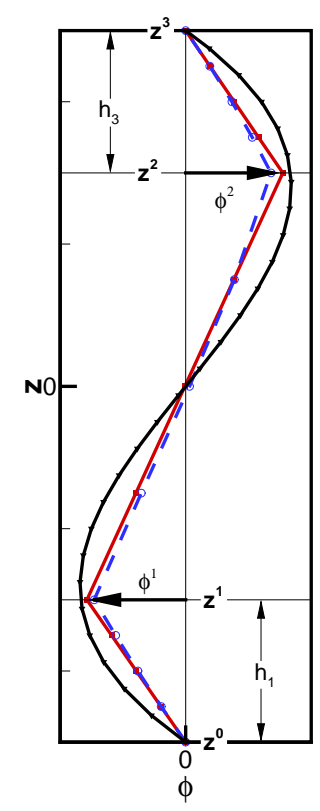

(a)

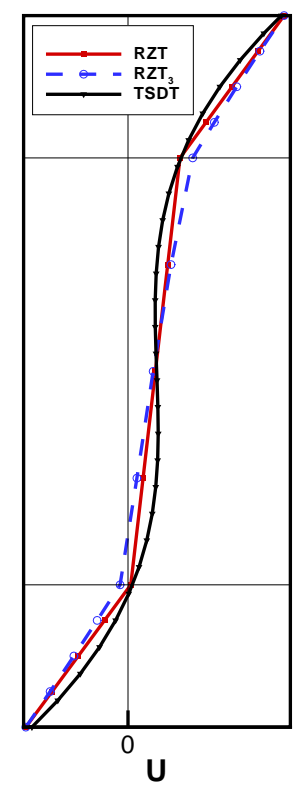

(b)

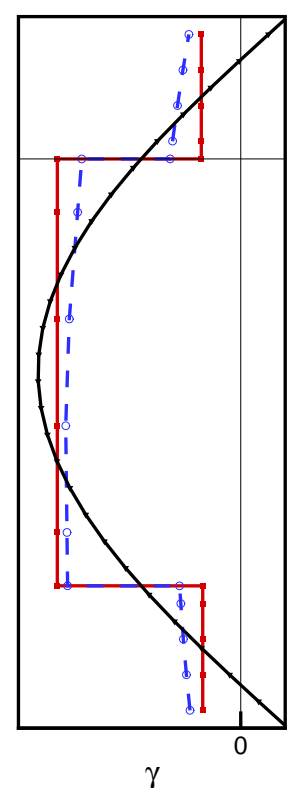

(c)

Figure 3: Additional displacements. (a) $\phi_{\alpha}$ function (b) total in-plane displacement $U_{\alpha}$ (c) total shear strain $\gamma_{\alpha}$

This approach may be associated to a modified version of the TSDT. The TSDT[32] pertains to HOT and in its original form requires $C^{1}$ approximations. For the unified formulation proposed in this work, this approach is modified and rewritten considering the standard kinematics of FSDT and the compatibility with TSDT. Such compatibility is achieved after equaling the sum of shear strains and/or the displacements at free top and bottom layer surfaces (see Appendix). This third function leads to a continuous transverse shear strain that nullifies at the external surfaces only if $\eta_{\alpha}$ vanishes.

None of the approximations ensures meeting of the IC condition, nor of the stress boundary conditions at surfaces. In the $\mathrm{RZT}_{(3)}$ approach both conditions are satisfied if the strain measure $\eta_{\alpha}$ vanishes. For homogeneous sections the RZT functions as defined above are null but a simple technique allows to obtain non-trivial functions[29]; in contrast the trigonometric approach is independent of the material. It must be noted that the implementation presented here allows to easily include other through the thickness interpolation functions with a minimum cost of additional codification.

Figure 3.a shows the different $\phi$ functions considered here for a three layer laminate, while 3.b-c show a possible configuration of in-plane displacements and shear strains resulting from the sum of the linear interpolation 
in (1) and the additional displacements in (40). Remind that the additional displacements are only in the in-plane directions $\left(Y_{1}, Y_{2}\right)$ of the convective system and note that as they are hierarchical displacements they do not interfere with rigid body motions. In contrast the base displacements are in the global Cartesian system and must be kept in such system to preclude problems with rigid body motions, especially if the focus is on geometrically non-linear problems.

The additional displacements lead to additional strains in both in-plane and transverse shear components as described next.

\subsection{In-plane strains}

The total in-plane strains (the expression below includes the transverse normal strain $E_{33}$ that has an important influence into the in-plane stresses) results the sum of the different contributions:

$$
\begin{aligned}
{\left[\begin{array}{c}
\varepsilon_{11} \\
\varepsilon_{22} \\
2 \varepsilon_{12} \\
\varepsilon_{33}
\end{array}\right] } & =\left[\begin{array}{c}
\bar{E}_{11} \\
\bar{E}_{22} \\
2 \bar{E}_{12} \\
\bar{E}_{33}
\end{array}\right]_{z=0}+z\left[\begin{array}{c}
\chi_{11} \\
\chi_{22} \\
2 \chi_{12} \\
\alpha
\end{array}\right]+\left[\begin{array}{c}
\phi_{1} \psi_{1,1} \\
\phi_{2} \psi_{2,2} \\
\phi_{1} \psi_{1,2}+\phi_{2} \psi_{2,1} \\
0
\end{array}\right] \\
& =\left[\mathbf{1}_{4}, z \mathbf{1}_{4}, \boldsymbol{\Phi}\right]\left[\begin{array}{c}
\mathbf{E}_{m} \\
\boldsymbol{\chi} \\
\nabla \psi
\end{array}\right] \\
\boldsymbol{\varepsilon} & =\mathbf{S}_{p} \boldsymbol{\varepsilon}_{p}
\end{aligned}
$$

Where the symbol $\varepsilon_{\alpha \beta}$ has been used to denote the total strain, to emphasize that it is assumed small and can be computed as the sum of the different contributions. Above $\mathbf{1}_{4}$ is the identity matrix of order 4 and the additional strains have been expressed as the product of the matrix $\boldsymbol{\Phi}$ and array $\nabla \boldsymbol{\psi}$ defined respectively by

$$
\boldsymbol{\Phi}=\left[\begin{array}{cccc}
\phi_{1} & 0 & 0 & 0 \\
0 & \phi_{2} & 0 & 0 \\
0 & 0 & \phi_{1} & \phi_{2} \\
0 & 0 & 0 & 0
\end{array}\right] \quad \nabla \boldsymbol{\psi}=\left[\begin{array}{c}
\psi_{1,1} \\
\psi_{2,2} \\
\psi_{1,2} \\
\psi_{2,1}
\end{array}\right]
$$

Using for the additional in-plane strains a similar approximation to that used for the original in-plane strains (Equation 16) the gradient $\nabla \boldsymbol{\psi}$ is computed at each mid-side point and is then averaged at the center

$$
\bar{\psi}_{\alpha, \beta}=\frac{1}{A^{f}} \sum_{k=1}^{N F} J_{K} \psi_{\alpha, \beta}^{K}
$$


such that the matrix relating incremental additional strains with incremental additional displacements can be written as:

$$
\overline{\mathbf{B}}_{\phi}^{I}=\frac{1}{A^{f}} \sum_{K=1}^{N F} J_{K}\left[\begin{array}{cc}
N_{, 1}^{I} & \\
& N_{, 2}^{I} \\
N_{, 2}^{I} & N_{, 1}^{I}
\end{array}\right]^{K}
$$

that allows to define additional stabilization strains, necessary for the hexahedral element only:

$$
\psi_{1,2}^{K H}=\psi_{1,2}^{K}-\bar{\psi}_{1,2}
$$

\subsection{Transverse shear strains}

The total transverse shear strains are

$$
\begin{aligned}
{\left[\begin{array}{l}
2 \varepsilon_{13} \\
2 \varepsilon_{23}
\end{array}\right](z) } & =\left[\begin{array}{l}
\bar{C}_{13} \\
\bar{C}_{23}
\end{array}\right]+\left[\begin{array}{l}
\beta_{1} \psi_{1} \\
\beta_{2} \psi_{2}
\end{array}\right]=\left[\mathbf{1}_{2},\left[\begin{array}{ll}
\beta_{1} & \\
& \beta_{2}
\end{array}\right]\right]\left[\begin{array}{l}
\overline{\mathbf{c}}^{s} \\
\boldsymbol{\psi}
\end{array}\right] \\
& =\left[\mathbf{1}_{2}, \boldsymbol{\beta}\right]\left[\begin{array}{l}
\overline{\mathbf{c}}^{s} \\
\boldsymbol{\psi}
\end{array}\right] \\
\boldsymbol{\gamma}(z) & =\mathbf{S}_{t}^{k} \varepsilon_{t}
\end{aligned}
$$

where $\mathbf{1}_{2}$ is the order 2 identity matrix and a diagonal matrix $\boldsymbol{\beta}$ has been defined.

To avoid numerical locking, an ANS approach for $\boldsymbol{\psi}$ is considered, that is almost identical to that used for $\mathbf{C}^{s}$. The differences are that $\boldsymbol{\psi}$ does not vary along $z$ (such variation is implicit in $\boldsymbol{\beta}$ ) and that the definition of the component $\psi_{\alpha}$ is simpler than that of $C_{\alpha 3}$. Then tangent components of $\boldsymbol{\psi}$ computed at each mid side point (middle surface) are linearly interpolated as in (28).

\section{Equivalent elasticity matrices and stress measures}

For a linear elastic composite laminate the internal strain energy per unit of middle surface can be written as a quadratic form of the generalized strains defined in (50) and (55):

$$
\begin{aligned}
W & =\frac{1}{2} \int_{h}(\varepsilon \cdot \boldsymbol{\sigma}+\boldsymbol{\gamma} \cdot \boldsymbol{\tau}) d z \\
& =\frac{1}{2} \int_{h}\left(\varepsilon_{p}^{T} \mathbf{S}_{p}^{T} \mathbf{D}_{p} \mathbf{S}_{p} \boldsymbol{\varepsilon}_{p}+\varepsilon_{t}^{T} \mathbf{S}_{t}^{T} \mathbf{D}_{t} \mathbf{S}_{t} \varepsilon_{t}\right) d z \\
& =\frac{1}{2} \int_{h}\left(\boldsymbol{\varepsilon}_{p}^{T}\left[\begin{array}{c}
\mathbf{1}_{3} \\
z \mathbf{1}_{3} \\
\boldsymbol{\Phi}^{T}
\end{array}\right] \mathbf{D}_{p}\left[\mathbf{1}_{3}, z \mathbf{1}_{3}, \boldsymbol{\Phi}\right] \boldsymbol{\varepsilon}_{p}+\boldsymbol{\varepsilon}_{t}^{T}\left[\begin{array}{c}
\mathbf{1}_{2} \\
\boldsymbol{\beta}
\end{array}\right] \mathbf{D}_{t}\left[\mathbf{1}_{2}, \boldsymbol{\beta}\right] \varepsilon_{t}\right) d z
\end{aligned}
$$


that allows to obtain conjugated generalized stresses. The constitutive matrices $\mathbf{D}_{p}$ and $\mathbf{D}_{t}$ used above (that change from layer to layer) result from a standard full 3D elastic relation split into in-plane (including transverse normal strain) and transverse shear behavior. We can define for the shell in-plane components as follows

$$
\begin{aligned}
\left(\overline{\mathbf{D}}_{p}\right)_{12 \times 12} & =\int_{h}\left[\begin{array}{ccc}
\mathbf{D}_{p} & \mathbf{D}_{p} z & \mathbf{D}_{p} \boldsymbol{\phi} \\
& \mathbf{D}_{p} z^{2} & \mathbf{D}_{p} \boldsymbol{\phi} z \\
\text { sym. } & & \boldsymbol{\phi}^{T} \mathbf{D}_{p} \boldsymbol{\phi}
\end{array}\right] d z \\
& =\left[\begin{array}{ccc}
\left(\mathbf{D}_{p}^{11}\right)_{4 \times 4} & \left(\mathbf{D}_{p}^{12}\right)_{4 \times 4} & \left(\mathbf{D}_{p}^{13}\right)_{4 \times 4} \\
& \left(\mathbf{D}_{p}^{22}\right)_{4 \times 4} & \left(\mathbf{D}_{p}^{23}\right)_{4 \times 4} \\
\text { sym. } & & \left(\mathbf{D}_{p}^{33}\right)_{4 \times 4}
\end{array}\right]
\end{aligned}
$$

Using the notation $\bar{z}_{k}=\frac{1}{2}\left(z^{k+1}+z^{k}\right)$ and $\bar{\phi}_{k}=\frac{1}{2}\left(\phi^{k+1}+\phi^{k}\right)$, these integrals for the RZT case ( $\boldsymbol{\beta}$ constant in each layer $k$ ) result (with $N L$ the number of composite layers):

$$
\begin{gathered}
\overline{\mathbf{D}}_{p}^{11}=\sum_{k=1}^{N L} \mathbf{D}_{p}^{k} h_{k} \quad \overline{\mathbf{D}}_{p}^{12}=\sum_{k=1}^{N L} \mathbf{D}_{p}^{k} h_{k} \bar{z}_{k} \quad \overline{\mathbf{D}}_{p}^{13}=\sum_{k=1}^{N L} \mathbf{D}_{p}^{k} \overline{\boldsymbol{\phi}}_{k} h_{k} \\
\overline{\mathbf{D}}^{22} \sum_{k=1}^{N L} \mathbf{D}_{p}^{k}\left(\bar{z}_{k}^{2}+\frac{h_{k}^{2}}{12}\right) h_{k} \quad \overline{\mathbf{D}}_{p}^{23}=\sum_{k=1}^{N L} \mathbf{D}_{p}^{k}\left[\overline{\boldsymbol{\phi}}_{k} \bar{z}_{k}+\boldsymbol{\beta}^{k} \frac{h_{k}^{2}}{12}\right] h_{k} \\
\overline{\mathbf{D}}_{p}^{33}=\sum_{k=1}^{N L}\left[\overline{\boldsymbol{\phi}}_{k}^{T} \mathbf{D}_{p}^{k} \overline{\boldsymbol{\phi}}_{k}+\left(\boldsymbol{\beta}^{k}\right)^{T} \mathbf{D}_{p}^{k} \boldsymbol{\beta}^{k} \frac{h_{k}^{2}}{12}\right] h_{k}
\end{gathered}
$$

While for the transverse shear components

$$
\left(\overline{\mathbf{D}}_{t}\right)_{4 \times 4}=\int_{h}\left[\begin{array}{cc}
\mathbf{D}_{t} & \mathbf{D}_{t} \boldsymbol{\beta} \\
\operatorname{sym} & \boldsymbol{\beta} \mathbf{D}_{t} \boldsymbol{\beta}
\end{array}\right] d z=\left[\begin{array}{cc}
\mathbf{D}_{t}^{11} & \mathbf{D}_{t}^{12} \\
\operatorname{sym} & \mathbf{D}_{t}^{22}
\end{array}\right]
$$

Where

$$
\begin{array}{ll}
\overline{\mathbf{D}}_{t}^{11} & =\sum_{k=1}^{N L} \mathbf{D}_{t}^{k} h_{k} \quad \overline{\mathbf{D}}_{t}^{12}=\sum_{k=1}^{N L} \mathbf{D}_{t}^{k} \boldsymbol{\beta}^{k} h_{k} \\
\overline{\mathbf{D}}_{t}^{22} & =\sum_{k=1}^{N L} \boldsymbol{\beta}^{k} \mathbf{D}_{t}^{k} \boldsymbol{\beta}^{k} h_{k}
\end{array}
$$

Thus the integrated forces and moments can be written as;

$$
\left[\begin{array}{c}
\left(\boldsymbol{\sigma}_{p}\right)_{12 \times 1} \\
\left(\boldsymbol{\sigma}_{t}\right)_{4 \times 1}
\end{array}\right]_{16 \times 1}=\left[\begin{array}{cc}
\left(\overline{\mathbf{D}}_{p}\right)_{12 \times 12} & \mathbf{0} \\
\mathbf{0} & \left(\overline{\mathbf{D}}_{t}\right)_{4 \times 4}
\end{array}\right]_{16 \times 16}\left[\begin{array}{c}
\left(\boldsymbol{\varepsilon}_{p}\right)_{12 \times 1} \\
\left(\boldsymbol{\varepsilon}_{t}\right)_{4 \times 1}
\end{array}\right]_{16 \times 1}
$$


In a more detailed manner:

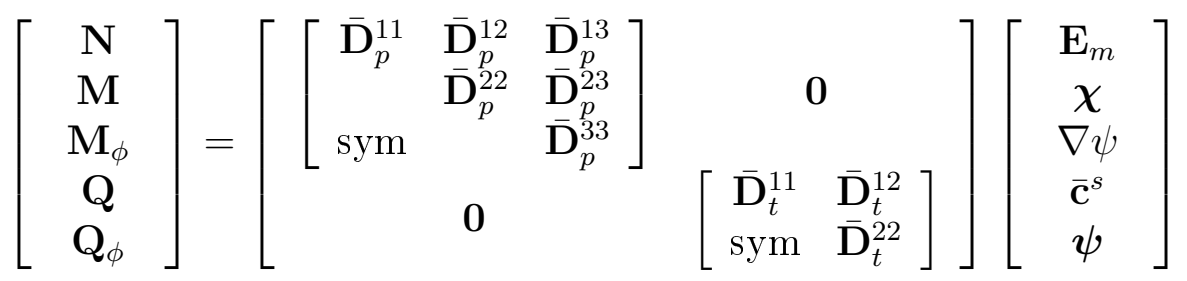

To emphasize the relation between stresses and integrated forces, note that $\mathbf{N}$ is:

$$
\begin{aligned}
\mathbf{N} & =\overline{\mathbf{D}}_{p}^{11} \mathbf{E}_{m}+\overline{\mathbf{D}}_{p}^{12} \boldsymbol{\chi}+\overline{\mathbf{D}}_{p}^{13} \nabla \boldsymbol{\psi} \\
& =\int_{h} \mathbf{D}_{p}\left(\mathbf{E}_{m}+z \boldsymbol{\chi}+\boldsymbol{\phi} \nabla \boldsymbol{\psi}\right) d z \\
& =\int_{h} \mathbf{D}_{p} \mathbf{E} d z=\int_{h} \boldsymbol{\sigma}_{p} d z
\end{aligned}
$$

and that $\mathbf{M}$ is:

$$
\begin{aligned}
\mathbf{M} & =\overline{\mathbf{D}}_{p}^{21} \mathbf{E}_{m}+\overline{\mathbf{D}}_{p}^{22} \boldsymbol{\chi}+\overline{\mathbf{D}}_{p}^{23} \nabla \boldsymbol{\psi} \\
& =\int_{h} \mathbf{D}_{p} z\left(\mathbf{E}_{m}+z \boldsymbol{\chi}+\boldsymbol{\phi} \nabla \boldsymbol{\psi}\right) d z \\
& =\int_{h} z \mathbf{D}_{p} \mathbf{E} d z=\int_{h} z \boldsymbol{\sigma}_{p} d z
\end{aligned}
$$

Note also that the first three components of $\mathbf{N}$ and $\mathbf{M}$ represent what is usually understood as membrane forces and bending moments in shell theories, while the fourth components in $\mathbf{N}$ and $\mathbf{M}$ do not appear in shell theories based upon the plane stress hypothesis. $N_{33}$ will be null if the plane stress condition were point-wise met while the fourth component in $\mathbf{M}$ is the balance equation for the internal DOF $\alpha$ of the EAS approach. Besides

$$
\begin{aligned}
\mathbf{Q} & =\overline{\mathbf{D}}_{t}^{11} \overline{\mathbf{c}}^{s}+\overline{\mathbf{D}}_{t}^{12} \boldsymbol{\psi} \\
& =\int_{h} \mathbf{D}_{t}\left(\overline{\mathbf{c}}^{s}+\boldsymbol{\beta} \boldsymbol{\psi}\right) d z=\int_{h} \boldsymbol{\tau} d z
\end{aligned}
$$

represents the transverse shear forces.

The in-plane gradient of the additional displacements leads to additional integrated moments

$$
\begin{aligned}
\mathbf{M}_{\phi} & =\overline{\mathbf{D}}_{p}^{31} \mathbf{E}_{m}+\overline{\mathbf{D}}_{p}^{32} \boldsymbol{\chi}+\overline{\mathbf{D}}_{p}^{33} \nabla \boldsymbol{\psi} \\
& =\int_{h} \mathbf{D}_{p} \boldsymbol{\phi}^{T}\left(\mathbf{E}_{m}+z \boldsymbol{\chi}+\boldsymbol{\phi}^{k} \nabla \boldsymbol{\psi}\right) d z \\
& =\int_{h} \boldsymbol{\phi}^{T} \mathbf{D}_{p} \mathbf{E} d z=\int_{h} \boldsymbol{\phi}^{T} \boldsymbol{\sigma}_{p} d z
\end{aligned}
$$


while due to the out-of-plane gradient of the additional displacements, additional transverse shear forces also appear:

$$
\begin{aligned}
\mathbf{Q}_{\phi} & =\overline{\mathbf{D}}_{t}^{21} \overline{\mathbf{c}}^{s}+\overline{\mathbf{D}}_{t}^{22} \boldsymbol{\psi} \\
& =\int_{h} \boldsymbol{\beta}^{T} \mathbf{D}_{t}\left(\overline{\mathbf{c}}^{s}+\boldsymbol{\beta} \boldsymbol{\psi}\right) d z=\int_{h} \boldsymbol{\beta}^{T} \boldsymbol{\tau} d z
\end{aligned}
$$

It must be emphasized that the strain measures $\left(\mathbf{E}_{m}, \boldsymbol{\chi}, \nabla \boldsymbol{\psi}\right)$ are associated with the middle surface and are constant in the element (one in-plane integration point is considered). But the transverse shear strains $\overline{\mathbf{c}}^{s}$ are linear across the thickness and although $\boldsymbol{\psi}$ are associated to the middle surface, the different slopes $\beta_{\alpha}^{k}$ at each layer lead to different transverse shear strains across the thickness, that is evidenced in the definition of matrix $\overline{\mathbf{D}}_{t}^{I J}$.

\section{Examples}

The examples below are mainly intended to show that the results of plate cases converge to analytical or numerical results reported in the literature for the RZT , i.e. the objective is to validate the implementation in solidshell finite elements. They are not intended to assess the RZT by comparing profiles of displacements and stresses across the thickness of the laminate, as this has already been extensively done by other authors. Besides, when nonlinear geometric analysis of sandwich curved shells is considered, comparisons are made against numerical models discretized with solid finite elements. In that case those examples are intended to show that the deformed configurations are broadly similar in both models but no comparison of stresses or displacements profiles across the thickness of the laminate are intended. The two solid-shell elements presented above are respectively denoted by $\mathrm{SH}$ (hexahedron) and SP (triangular prism). For numerical comparisons, results obtained with shell elements combined with the RZT as shown in [8] are used, these are a four-node quadrilateral (denoted here as SQ) and a linearly interpolated six-node triangle (denoted here as ST). Also the eight-node solid element presented in [11], denoted here as S8, is used. This solid element uses an ANS approach for the transverse shear strains that allows to consider elements with a high aspect ratio between the in-plane and the normal directions. Element S8 can then be used to model shell structures including two or more elements across the thickness.

The transverse sections considered in the examples are mainly sandwich laminates although a angle-ply laminate is also taken into account. So three types of materials are involved, those to be used as external layers, those to be used as the core of sandwich sections and an orthotropic lamina for angle-ply 
assemblies. Tables 1-2 show the properties of these material, that include four stiff materials $(1,2,6$ and 7$)$ used as external walls of sandwich sections, four soft materials $(4,5,8$ and 9$)$ used as the core part of sandwich sections and an orthotropic lamina (3). Three symmetric and two asymmetric sections are considered. Table 3 shows the stacking sequence of the symmetric sandwich sections, all of them with 5 layers, where $\alpha$ (in degrees) indicates the angle of material direction 1 with local laminate direction 1. The Table 4 shows the stacking sequence of the two asymmetric sections, a sandwich one made with isotropic materials and an angle ply.

\begin{tabular}{|c|r|r|r|r|r|r|r|r|r|}
\hline Mat & $E_{1}$ & $E_{2}$ & $E_{3}$ & $\nu_{12}$ & $\nu_{13}$ & $\nu_{23}$ & $G_{12}$ & $G_{13}$ & $G_{23}$ \\
\hline \hline 1 & 50 & 10 & 10 & 0.05 & 0.05 & 0.25 & 5 & 5 & 5 \\
\hline 2 & 131 & 10.34 & 10.34 & 0.22 & 0.22 & 0.49 & 6.895 & 6.205 & 6.895 \\
\hline 3 & 20 & 1.0 & 0.833 & 0.25 & 0.00 & 0.00 & 0.6 & 0.6 & 0.5 \\
\hline 4 & $10^{-5}$ & $10^{-5}$ & 0.07585 & 0.01 & 0.01 & 0.01 & 0.0225 & 0.0225 & 0.0225 \\
\hline 5 & 0.01 & 0.01 & 0.07585 & 0.01 & 0.01 & 0.01 & 0.0225 & 0.0225 & 0.0225 \\
\hline
\end{tabular}

Table 1: Orthotropic material properties used in the laminates ( $E_{I}$ and $G_{I J}$ in $\mathrm{GPa}$ )

\begin{tabular}{|c|r|r|}
\hline Mat & $E$ & $\nu$ \\
\hline \hline 6 & 730 & 0.25 \\
\hline 7 & 219 & 0.25 \\
\hline 8 & 0.00689 & 0.00 \\
\hline 9 & 0.730 & 0.25 \\
\hline
\end{tabular}

Table 2: Isotropic material properties used in the laminates ( $E$ in GPa)

\begin{tabular}{|r|r|r|}
\hline Mat & $h_{k}[\%]$ & $\alpha$ \\
\hline 1 & 5 & 0 \\
\hline 1 & 5 & 90 \\
\hline 4 & 80 & 0 \\
\hline 1 & 5 & 90 \\
\hline 1 & 5 & 0 \\
\hline
\end{tabular}

(1)

\begin{tabular}{|r|r|r|}
\hline Mat & $h_{k}[\%]$ & $\alpha$ \\
\hline 1 & 5 & 0 \\
\hline 1 & 5 & 90 \\
\hline 5 & 80 & 0 \\
\hline 1 & 5 & 90 \\
\hline 1 & 5 & 0 \\
\hline
\end{tabular}

(2)

\begin{tabular}{|r|r|r|}
\hline Mat & $h_{k}[\%]$ & $\alpha$ \\
\hline 2 & 4.1667 & 0 \\
\hline 2 & 4.1666 & 90 \\
\hline 6 & 83.334 & 0 \\
\hline 2 & 4.1666 & 90 \\
\hline 2 & 4.1667 & 0 \\
\hline
\end{tabular}

(3)

Table 3: Symmetric laminates stacking sequence.

\subsection{Cantilever beam under end point load}

A simple cantilever beam under a point load is initially considered. Although this is not the focus of this element, allows a detailed comparison of 


\begin{tabular}{|r|r|}
\hline Mat & $h_{k}[\%]$ \\
\hline 6 & 10 \\
\hline 9 & 80 \\
\hline 7 & 10 \\
\hline
\end{tabular}

(4)

\begin{tabular}{|r|r|r|}
\hline Mat & $h_{k}[\%]$ & $\alpha$ \\
\hline 3 & 25 & 0 \\
\hline 3 & 25 & 30 \\
\hline 3 & 25 & -30 \\
\hline 3 & 25 & 90 \\
\hline
\end{tabular}

$(5)$

Table 4: Asymmetric laminates stacking sequence.

the results obtained with an expensive solid model and the present formulation. This example for a wide range of aspect ratios has been analyzed in [21]. Here the geometric parameters considered are: length $L=200 \mathrm{~mm}$, section width $b=40 \mathrm{~mm}$ and thickness $h=20 \mathrm{~mm}$, while the applied load is $P=20 N$. The section used is number 4 , a three-layered asymmetric sandwich laminate (see Table 4), where the core is eight times thicker than each face sheet. This example was chosen because the results obtained with beam finite elements including the RZT approach are in excellent agreement with the analytical ones. In this case a solid finite element model using element S8 is used for comparison. It includes 20 equal elements along the length and four elements for each layer leading to 240 elements and 546 nodes. All the displacement DOFs are constrained at the root and, in order to avoid an ad hoc distribution of the point load over the 26 nodes at the end section, all the nodes at the free end are constrained to displace by the same value in the normal direction (leading to $u_{z}^{L}=0.027888 \mathrm{~mm}$ ). For the present formulation (RZT $_{(3)}$ is used), 20 equal elements are also considered in the discretization and the same constraints of the solid model are applied at the root and at the loaded end ( $u_{z}^{L}=0.027877 \mathrm{~mm}$ is obtained in this case). The difference in the tip displacement is less than $0.04 \%$. A detailed comparison of the results from both models is presented for the section at mid span, i.e. far from both boundaries. Figure 4.a shows a plot of the displacement profiles across the thickness. It can be seen that the axial displacements $\left(u_{1}\right)$ are practically coincident. The average transverse displacements $\left(u_{z}\right)$ are different. At the beam axis the displacement obtained with the solid model is $u_{z}^{S 8}=0.011234$ while with the present element it is slightly less $u_{z}^{S H}=0.011216$. For an easier comparison of the profiles in Figure 4.a, the results obtained with the solid model S8 are shifted -0.000018 to match the displacement of the SH model at the beam axis. It can be seen that in doing so the profiles are coincident. The quadratic variation of the $u_{z}^{S H}(z)$ is computed using equation (39). Figure 4.b plots the axial and transverse shear stresses. The axial stresses $\sigma_{11}(z)$ are almost coincident for both models. For the shear stress $\tau_{1 z}$ four curves are plotted: a) $\mathbf{S} 8-\tau_{1 z}$ is the value obtained with the solid model, it must be 


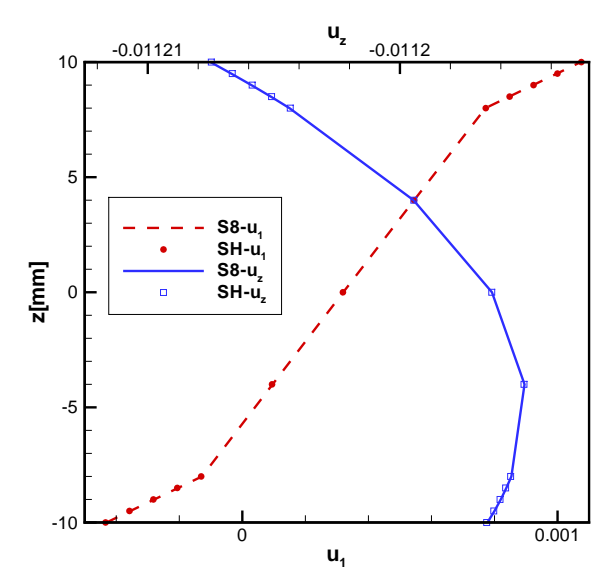

(a)

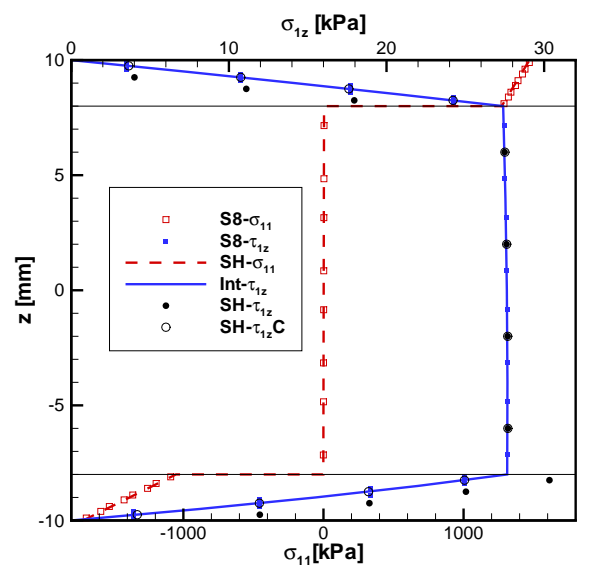

(b)

Figure 4: Three-layer cantilever beam. (a) Displacements (b) Stresses

noted that the ANS of element S8 leads to constant shear stress across the element thickness, for that reason, the same values appear at two different integration points; b) Int- $\tau_{1 z}$ is the value obtained integrating the equilibrium equation in $z$ direction from the axial stress $\mathrm{SH}-\sigma_{11}\left(Y_{1}, z\right)$; c) SH $-\tau_{1 z}$ is the value obtained consistently from the present element kinematics using the constitutive equation at each layer; and d) $\mathrm{SH}-\tau_{1 z} \mathrm{C}$ is the latter's continuous component $\bar{\tau}_{1 z}^{k}$ (see equation 42 ). The excellent correlation between $\mathrm{S} 8-\tau_{1 z}$ and Int- $\tau_{1 z}$ is a consequence of the excellent correlation in $\sigma_{11}$. It is worth noting that the continuous part $\mathrm{SH}-\tau_{1 z} \mathrm{C}$ is also coincident with those results. As the whole $\mathrm{RZT}_{(3)}$ formulation does not meet the boundary conditions at the external surfaces, the curve $\mathrm{SH}-\tau_{1 z}$ over predicts shear stresses at the bottom layer and under predicts shear stresses at the top layer.

\subsection{Simply supported square plate under sinusoidal load}

The second example considered is a simple supported square plate under sinusoidal load for which an analytical solution based on the FSDT plus the RZT was given in [13]. The transverse section corresponds to sandwich laminate 1 with a total thickness of $t=0.5 \mathrm{~m}$. The side of the square plate is $a=10 \mathrm{~m}$ so the aspect ratio is $a / h=20$. The transverse load, applied at the top surface, has a sinusoidal variation in both in-plane directions with a maximum amplitude $q_{0}=1 \mathrm{kPa}$ at the center and null at the simple supported sides.

One quarter of the plate is discretized due to double symmetry with the center of the plate located at the origin of the coordinate system. The simply supported boundary condition implies that the nodes on the upper surface 


\begin{tabular}{|c|r|r|r|r|r|r|r|}
\hline & Ref. & SH+RZT & SP+RZT & SQ+RZT & ST+RZT & FSDT & CLPT \\
\hline \hline$u_{z}$ & 0.6742 & 0.6722 & 0.6738 & 0.6740 & 0.6752 & 0.2472 & 0.2350 \\
\hline$u_{z}[\%]$ & 100.00 & 99.70 & 99.94 & 99.97 & 100.14 & 36.67 & 34.85 \\
\hline
\end{tabular}

Table 5: Simple supported square plate under sinusoidal load

$(T)$ have identical displacements to the nodes on the lower surface $(B)$ but with the opposite sign:

\begin{tabular}{|c|c|c|c|c|c|c|c|c|}
\hline Boundary & $u_{1}^{B}$ & $u_{2}^{B}$ & $u_{z}^{B}$ & $u_{1}^{T}$ & $u_{2}^{T}$ & $u_{z}^{T}$ & $\psi_{1}$ & $\psi_{2}$ \\
\hline \hline$Y_{1}=0$ & 0.0 & & & 0.0 & & & 0.0 & \\
\hline$Y_{2}=0$ & & 0.0 & & & 0.0 & & & 0.0 \\
\hline$Y_{1}=a / 2$ & & & & $-u_{1}^{B}$ & $-u_{2}^{B}$ & $-u_{z}^{B}$ & & 0.0 \\
\hline$Y_{2}=a / 2$ & & & & $-u_{1}^{B}$ & $-u_{2}^{B}$ & $-u_{z}^{B}$ & 0.0 & \\
\hline
\end{tabular}

A structured mesh, with a uniform increment in both directions of $\Delta=$ $0.15625 \mathrm{~m}$ has been considered, that implies 33 nodes per side, 1089 nodes over each surface and 1024 hexahedral 8-node elements ( $\mathrm{SH}$ ) or 2048 prismatic 6-node elements (SP) and 8259 degrees of freedom (DOFs). Due to the sandwich-type properties of the laminate, the RZT interpolation has been considered.

The normal displacement at the plate center indicated in the Reference [13] is

$$
u_{z}(0,0)=0.1118 \times \frac{D_{11}}{a^{4}} q_{0}=0.6742 m m
$$

Table 5 shows the displacement of the central point (average of upper and lower surface) obtained with both elements developed here and other models for comparison purposes. The results show a very good correlation between shell and solid-shell elements enhanced with the RZT kinematic in both cases. Figure 5 illustrates the amplitude of the additional displacements for the two solid-shell elements presented here.

\subsection{Clamped square plate under uniform load}

The same geometry of previous example but under uniform load $q=$ $1 \mathrm{KPa}$ and clamped boundary was also considered. In this case the comparison is made against a finite element model of standard 20-node solid elements (S20) with a mesh of $16 \times 16 \times 9$ elements (one element per layer for the outer layers and five elements for the core). For the latter model, used as a reference, the displacement of the center of the plate is $w_{\max }^{S 20}=0.6936$ (average between bottom and top surfaces). The results in Table 6 show a very good correlation between the solid, the solid-shell and shell models. Note the very low values predicted by standard shell theories FSDT and CLPT. 


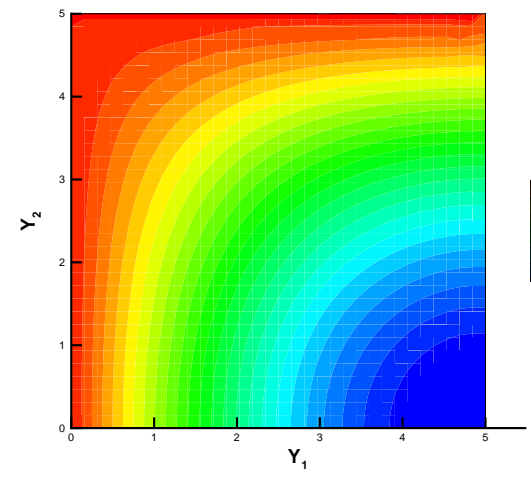

(a)

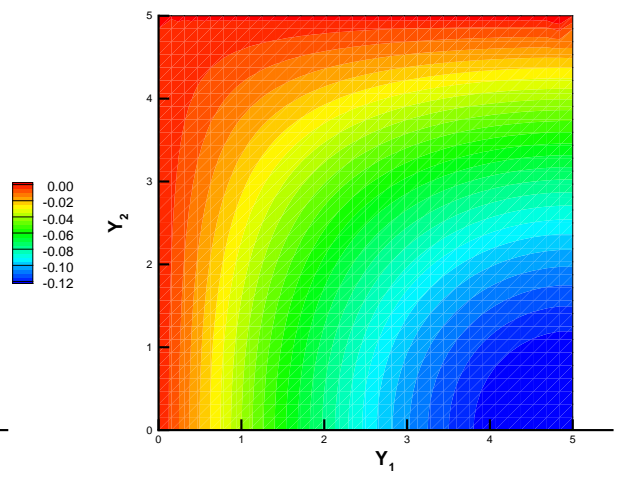

(b)

Figure 5: Amplitude of the additional displacement in $Y_{1}$ direction $\left(\psi_{1}\right)$. (a) SH element (b) SP element

\begin{tabular}{|c|r|r|r|r|r|r|r|}
\hline & S20 & SH+RZT & SP+RZT & SQ+RZT & ST+RZT & FSDT & CLPT \\
\hline \hline$u_{z}$ & 0.6936 & 0.6881 & 0.6912 & 0.6916 & 0.6985 & 0.1134 & 0.0954 \\
\hline$u_{z}[\%]$ & 100.00 & 99.21 & 99.65 & 99.71 & 100.71 & 16.35 & 13.75 \\
\hline
\end{tabular}

Table 6: Clamped square plate under uniform load

\subsection{Natural frequencies of a clamped-free cylindrical panel}

This example is intended to assess the importance of the additional displacements for the global response of the structure when the section is an angle-ply laminate. The geometry of the shallow cylindrical panel is shown in Figure 6. The length is $L=300 \mathrm{~mm}$, the radius is $R=1000 \mathrm{~mm}$, and the subtended angle is $\alpha=0.2$. The thickness of the panel is $h=10 \mathrm{~mm}$, leading to an aspect ratio $\alpha R / h=20$. The panel is clamped along the curved sides and free along the straight sides. The section considered is the asymmetric four-layer angle-ply laminate 5 (see Table 4 ). It must be noted that lumped mass matrices (with a mass density $\rho=2000 \frac{\mathrm{kg}}{\mathrm{m}^{3}}$ ) were considered in the analysis leading to different rotatory inertia in shell and solid-shell models. For all the models, the mid-surface is discretized with 60 elements along the cylinder axis and 40 elements along the parallel. The other finite element models used for comparison are the shell element SQ with and without RZT and a solid model including eight S8 elements across the thickness (2 elements per ply). Table 7 shows the first twelve natural frequencies $f$ for the different numerical models beginning with the values obtained with the solid model (S8) $f^{S 8}$. For an easier comparison the table is completed with the percentage differences with respect to the solid model frequency, i.e. $100 \times\left(\frac{f}{f^{S 8}}-1\right)$. The 


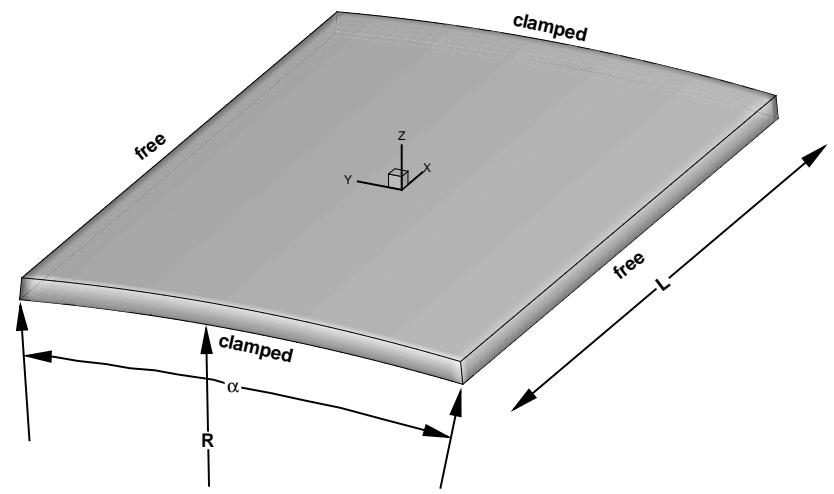

Figure 6: Clamped-free cylindrical panel

angle-ply laminate does no lead to high ratios between the shear modulus of the different layers as for sandwich shells $\left(G_{13} / G_{23}=1.2\right.$ in this case). That is why the shell stiffness (and consequently the natural frequencies) does no differ very much between the alternative numerical models. The percentage differences between present element and the solid model are generally not greater than the differences between the shell model and the solid model. Finally the vibration modes associated with natural frequencies 9-12 are plotted in Figure 7, where the contour-fill of the component along the global $X_{3}=Z$ coordinate is also shown. The vibration modes obtained are almost identical to those obtained with the solid model (not shown).

\begin{tabular}{|r|r|r|r|r|r|r|}
\hline Mode & S8 & SQ+RZT & SH+RZT & SH+RZT3 & SH+TRIG & FSDT \\
\hline \hline 1 & 42.12 & 0.776 & 0.450 & 0.499 & 0.331 & 0.714 \\
\hline 2 & 45.24 & 0.401 & 0.328 & 0.101 & -0.109 & 0.288 \\
\hline 3 & 87.13 & 0.207 & -0.580 & -0.724 & -0.763 & -0.039 \\
\hline 4 & 107.80 & 1.276 & 0.743 & 0.828 & 0.475 & 1.148 \\
\hline 5 & 111.64 & 0.906 & 0.547 & 0.371 & -0.004 & 0.729 \\
\hline 6 & 146.49 & 0.791 & -0.247 & -0.599 & -0.800 & 0.424 \\
\hline 7 & 183.72 & 0.406 & -1.415 & -1.490 & -1.401 & -0.100 \\
\hline 8 & 197.34 & 1.710 & 0.920 & 1.055 & 0.512 & 1.500 \\
\hline 9 & 202.01 & 1.413 & 0.728 & 0.633 & 0.068 & 1.161 \\
\hline 10 & 222.79 & 0.652 & -1.301 & -1.679 & -1.711 & 0.031 \\
\hline 11 & 234.68 & 1.330 & 0.009 & -0.355 & -0.762 & 0.877 \\
\hline 12 & 294.60 & 1.140 & -0.981 & -1.563 & -1.766 & 0.426 \\
\hline
\end{tabular}

Table 7: Clamped-free cylindrical panel. Natural frequencies and percentage differences 

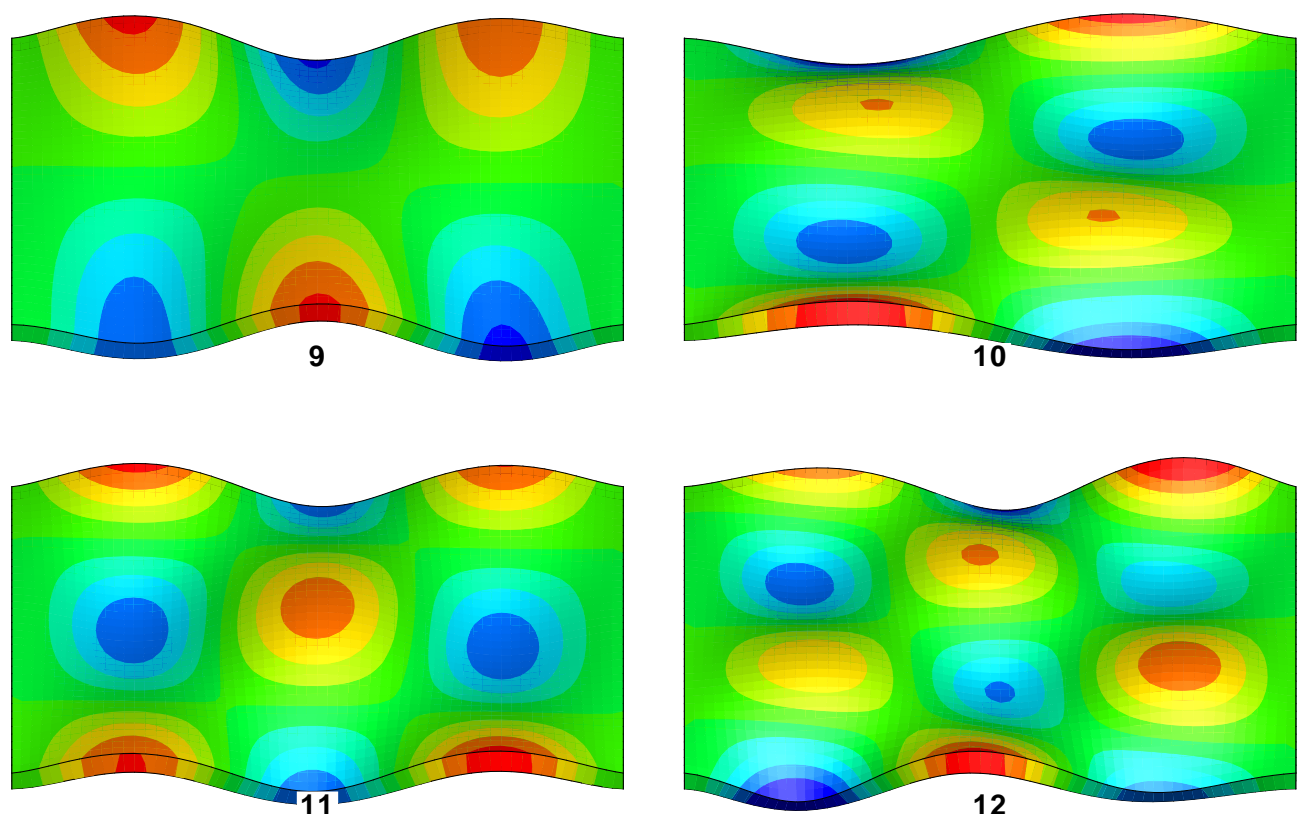

Figure 7: Clamped-free cylindrical panel. Vibration modes 9-12

\subsection{Spherical sector with line loads}

To test the present formulation in double curved shells with geometric non-linearities, a simple spherical sector as shown in Figure 8 with radius $R=10 \mathrm{~m}$ and an angle $\alpha=30^{\circ}$ was analyzed. The applied loads are uniform line loads parallel to the $X-Y$ plane (see the orientation axis in the figure), outward along meridian $\mathrm{A}-\mathrm{A}(+X)$ and $\mathrm{C}-\mathrm{C}(-X)$ and inward along meridian $\mathrm{B}-\mathrm{B}(-Y)$ and $\mathrm{D}-\mathrm{D}(+Y)$.

The section considered is number 2 with a total thickness $t=0.2 \mathrm{~m}$. The reference line load $q_{0}$ is $1 M N / m$. The finite element discretization covers one fourth of the geometry with 40 divisions in the parallel direction and 13 along the meridian, leading to 1230 nodes and 520 hexahedral elements or 1040 prismatic elements. In this case comparisons are made against a 3D solid element model (S8) with the same surface discretization but 6 element layers across the thickness.

The maximum inward displacement considered is $3 \mathrm{~m}$ ( $30 \%$ of the radius). Figure 9 plots the inward (larger) and outward displacements of the loaded points of the free lower border (average between top and bottom surfaces). The results obtained with solid elements (S8) are assumed as "target values", but the results obtained with shell elements (SQ with RZT) are also included for comparison. The results obtained with the prismatic element including 


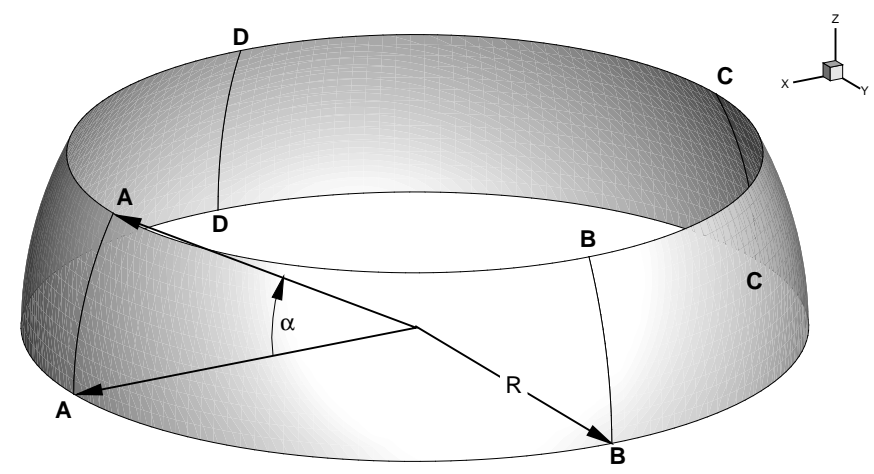

Figure 8: Spherical sector with line loads

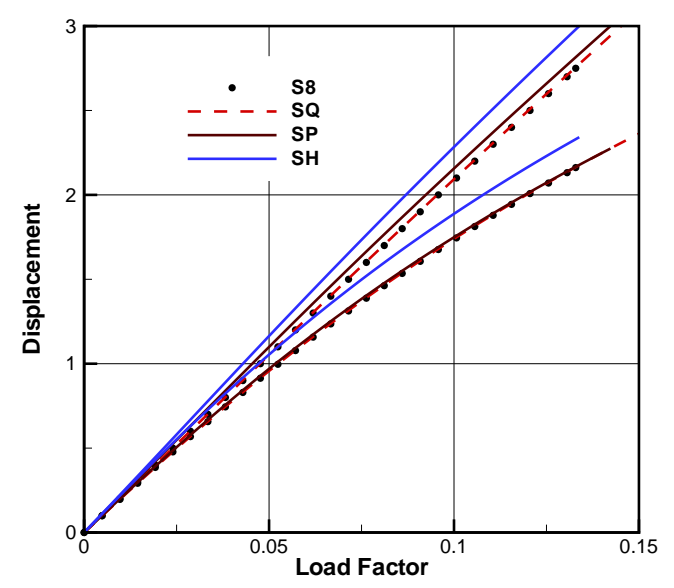

Figure 9: Spherical sector with line loads

the RZT kinematic (SP) show a very good correlation with those obtained with both shell elements and solid elements. The hexahedral element including the RZT kinematic $(\mathrm{SH})$ results show a more flexible behavior that may be due to the reduced integration.

\subsection{Buckling of a cylindrical shell}

This example is intended to study the stability of a clamped cylindrical shell under axial and external pressure loads. The geometrical parameters of the cylinder are radius $R=10 \mathrm{~m}$, length $L=20 \mathrm{~m}$ and thickness $t=0.25 \mathrm{~m}$ while the transverse section is defined by the laminate 2 where the stiffer direction is associated with the cylinder axis. Only one eighth of the total cylinder has been discretized (one quadrant and half the length) enforcing symmetry conditions on three sides and a clamped boundary condition at the 
remaining side. The objective of these assumptions, that arbitrary restrict the bifurcation modes to these symmetries, is to have a manageable solid (S8) finite element model for comparison purposes. The discretization with $\mathrm{SH}$ elements includes 5246 nodes and 2520 elements resulting from a structured mesh with 60 equal divisions along the quadrant considered and 42 divisions along half the cylinder length. As the transverse section is of the sandwich type, the RZT interpolation is used for the additional in-plane DOFs. The solid model used for comparison has the same discretization of the middle surface and 7 elements across the thickness.

Table 8 shows the critical loads for both axial load and external pressure for the different numerical models. A very good correlation can be seen between the results obtained with the solid model, the shell model (SQ+RZT) and the solid-shell models presented here. The Table also includes for reference the buckling loads obtained using the CLPT and the FSDT with the isotropic shear factor $5 / 6$.

\begin{tabular}{|r|r|r|}
\hline Model & Axial Load $[\mathrm{MN} / \mathrm{m}]$ & External Pressure [MPa] \\
\hline \hline S8 & 7.040 & 0.3888 \\
\hline SQ+RZT & 7.093 & 0.3988 \\
\hline SH+RZT & 6.983 & 0.4056 \\
\hline SP+RZT & 7.221 & 0.4053 \\
\hline FSDT & 20.529 & 0.6583 \\
\hline CLPT & 20.614 & 0.6616 \\
\hline
\end{tabular}

Table 8: Critical loads

Figure 10 shows the buckling modes due to axial load while Figure 11 shows those due to external pressure. It can be seen that not only the critical loads are similar, but the buckling modes are also similar for the different models considered. In contrast the buckling modes obtained using the CLPT or the FSDT $(\mathrm{SCF}=5 / 6$ ) lead to different buckling modes (not shown).

\section{Conclusions}

A general formulation for the mechanical analysis of composite laminated structures with large displacements is proposed in this work. The model employs solid-shell finite elements and considers the RZT and a modified TSDT in a unified way. The elements considered are a tri-linear hexahedron and a 6-node triangular prism. Both elements use as strain measure a modified right Cauchy-Green deformation tensor, where 5 (those associated with the in-plane and transverse shear strains) of its 6 components are computed at 


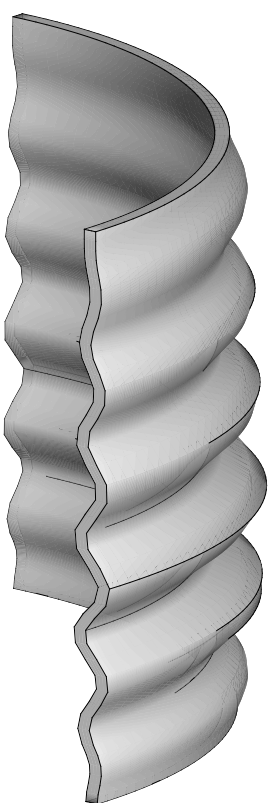

(a)

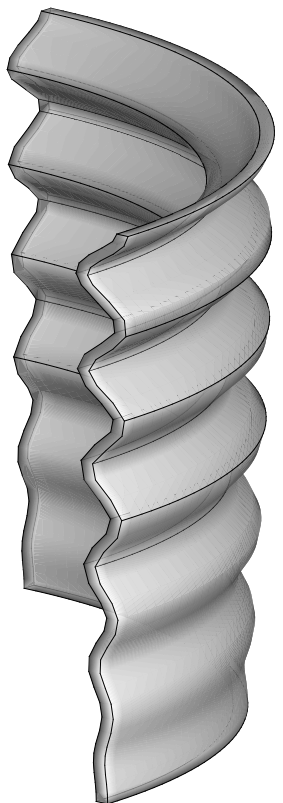

(b)

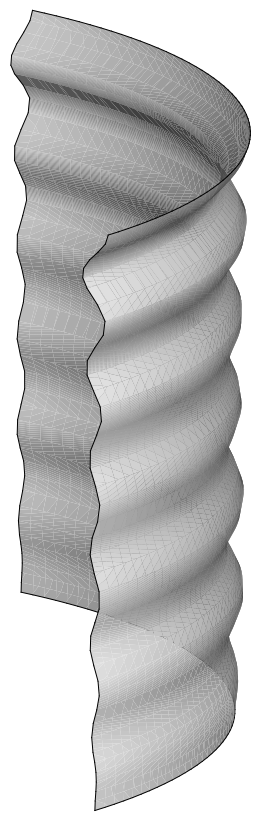

(c)

Figure 10: Buckling mode under axial load (a) S8; (b) SH; (c) SQ

the center of both the lower and upper surfaces using assumed strain techniques and are linearly interpolated across the element thickness. The sixth component (transverse normal strain) is computed at the element center and enhanced with an additional degree of freedom using the enhanced assumed strain (EAS) technique.

The main aspects of the implementation are:

- The additional displacements are hierarchical displacement components in a convective local systems defined over the shell middle surface.

- The constitutive models are restricted to small elastic deformations thus making it unnecessary to distinguish between different strain measures.

- The strains are computed as the sum of those due to changes in the element configuration plus those due to the hierarchical displacements.

- The ANS approach used for the transverse shear strains in the original elements is also used for the additional shear strains. 


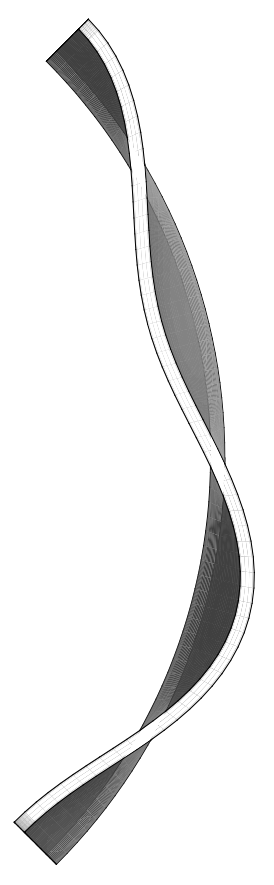

(a)

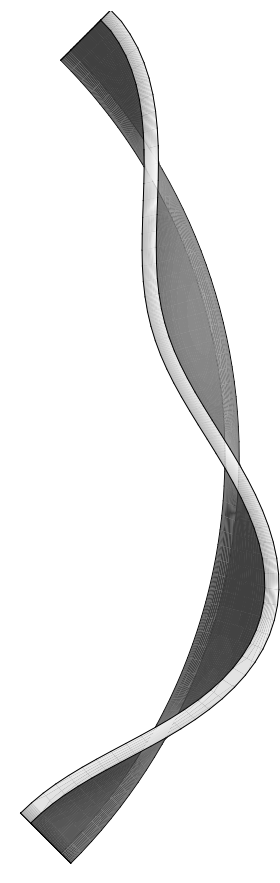

(b)

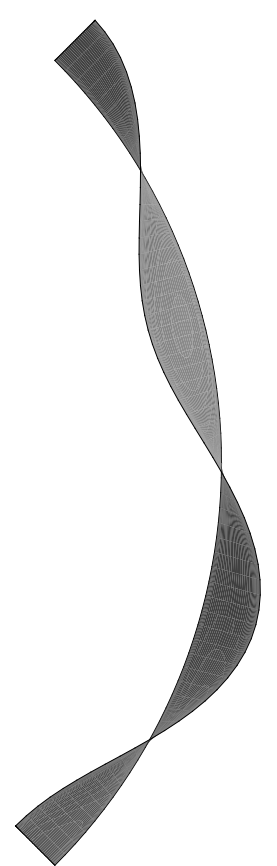

(c)

Figure 11: Buckling mode under external pressure (a) S8; (b) SH; (c) SQ

- For the hexahedral element, the stabilization schemes used for the additional strains are similar to those used in the original element, for both the in-plane and transverse shear strains.

- A lumped mass matrix has been used for the computation of natural frequencies.

The main conclusions that can be drawn are:

- For the linear examples considered, both static equilibrium analysis and natural frequencies computation, the elements presented here lead to results similar to those obtained with shell elements based on a seven parameter approach (5 from the FSDT plus 2 hierarchical DOFs)

- For double curved shells with geometrical non-linearities, the comparisons with solid elements show a very good correlation.

- Linear buckling analysis of cylindrical shell shows an excellent correlation between solid and solid-shell models for both critical loads and buckling modes. It also shows the strong limitations of classical approximations (FSDT and CLPT) for sandwich shells. 
- The ANS used for transverse shear strains avoids the numerical locking. The stabilization schemes for the reduced integrated SH element also work properly.

\section{Acknowledgment}

First author acknowledges the financial support from CONICET (Argentina) and SeCyT-UNC. This work has also been supported by European Research Council through of Advanced Grant: ERC-2012-AdG 320815 COMP-DES-MAT "Advanced tools for computational design of engineering materials", by the Spanish Ministerio de Economía y Competividad through the project: MAT2014-60647-R "Multi-scale and multi-objective optimization of composite laminate structures (OMMC)", and by International Center for Numerical Method in Engineering (CIMNE). All this support is gratefully acknowledged.

\section{References}

[1] A. Barut and A. Madenci, E.and Tessler. C0-continuous triangular plate element for laminated composite and sandwich plates using the $\{2,2\}$ refined zigzag theory. Composite Structures, 106:835-853, 2013.

[2] E. Carrera. Historical review of zig-zag theories for multilayered plates and shells. Applied Mechanics Reviews, 56:298-308, 2003.

[3] M. Di Sciuva, M. Gherlone, M. Iurlaro, and A. Tessler. A class of higherorder c0 composite and sandwich beam elements based on the refined zigzag theory. Composite Structures, 132:784-803, 2015.

[4] A. Eijo, E. Oñate, and S. Oller. A four-noded quadrilateral element for composite laminated plates/shells using the refined zigzag theory. International Journal for Numerical Methods in Engineering, 95:631660, 2013.

[5] A.J.M. Ferreira, C.M.C. Roque, and R.M.N. Jorge. Analysis of composite plates by trigonometric shear deformation theory and multiquadrics. Computers \& Structures, 27:2225-2237, 2005.

[6] M. Filippi and E. Carrera. Bending and vibration analyses of laminated beams by using a zig-zag-layer-wise theory. Composites Part B, 98:269$280,2016$. 
[7] F.G. Flores. Development of a non-linear triangular prism solid-shell element using ans and eas techniques. Computer Methods in Applied Mechanics and Engineering, 266:81-97, 2013.

[8] F.G. Flores. Implementation of the refined zigzag theory in shell elements with large displacements and rotations. Composite Structures, 118:560-570, 2014.

[9] F.G. Flores. A simple reduced integration hexahedral solid-shell element for large strains. Computer Methods in Applied Mechanics and Engineering, 303:260-287, 2016.

[10] F.G. Flores and C.F. Estrada. A rotation free thin shell quadrilateral. Computer Methods in Applied Mechanics and Engineering, 196:26312646, 2007.

[11] F.G. Flores and E. Oñate. Un elemento de sólido con una mejora en el comportamiento del corte transversal para el tratamiento de láminas. Revista Internacional de Métodos Numéricos para Cálculo y Diseño en Ingeniería, 27(4):256-268, 2011.

[12] M. Gherlone, A. Tessler, and Di Sciuva M. C0 beam element based on the refined zigzag theory for multilayered composite and sandwich laminates. Composite Structures, 93:2882-2894, 2011.

[13] L. Iurlaro, M. Gherlone, M. Di Sciuva, and Tessler. Assessment of the refined zigzag theory for bending, vibration, and buckling of sandwich plates: a comparative study of different theories. Composite Structures, 106:777-792, 2013.

[14] L. Iurlaro, M. Gherlone, M. Di Sciuva, and Tessler. Refined zigzag theory for laminated composite and sandwich plates derived from reissner's mixed variational theorem. Composite Structures, 133:809-817, 2015.

[15] L. Iurlaro, M. Gherlone, M. Di Sciuva, and A. Tessler. The (3,2)-mixed refined zigzag theory for generally laminated beams: Theoretical development and c0 finite element formulation. Solids and Structures, 73-74:1-19, 2015.

[16] M. Karama, K.S. Afaq, and S. Mistou. A new theory for laminated composite plates. Journal of Material Design and Applications, 223(2):5362,2009 . 
[17] J.L. Mantari, A.S. Oktem, and C. Guedes Soares. A new trigonometric shear deformation theory for isotropic, laminated composite and sandwich plates. International Journal of Solids and Structures, 49(1):43-53, 2012.

[18] X. Martinez, F. Rastellini, S. Oller, F. Flores, and E. Oñate. Computationally optimized formulation for the simulation of composite materials and delamination failures. Journal of Composites part B, 42:134-144, 2011.

[19] H. Matsunaga. Assessment of a global higher-order deformation theory for laminated composite and sandwich plates. Composite Structures, 56:279-91, 2002.

[20] L.G. Nallim and S. Oller. An analytical - numerical approach to simulate the dynamic behaviour of arbitrarily laminated composite plate. Composite Structures, 85:311-325, 2008.

[21] L.G. Nallim, S. Oller, Oãte E., and Flores F. A hierarchical finite element for composite laminated beams using a refined zigzag theory. Composite Structures, 163:168-184, 2017.

[22] L.G. Nallim, S. Oller, and R.O. Grossi. Statical and dynamical behaviour of thin fibre reinforced composite laminates with different shapes. Computer Methods in Applied Mechanics and Engineering, 194:1797-1822, 2005.

[23] E. Oñate, A. Eijo, and S. Oller. Simple and accurate two-noded beam element for composite laminated beams using a refined zigzag theory. Computer Methods in Applied Mechanics and Engineering, 213-216:362382, 2012.

[24] R.F. Rango, L.G. Nallim, and S. Oller. Formulation of enriched macro elements using trigonometric shear deformation theory for free vibration analysis of symmetric laminated composite plate assemblies. Composite Structures, 119:38-49, 2015.

[25] R.F. Rango, L.G. Nallim, and S. Oller. An enriched macro finite element for the static analysis of thick general quadrilateral laminated composite plates. Mechanics of Advanced Materials and Structures, 23(10):11971206, 2016.

[26] J.N. Reddy. A simple higher-order theory for laminated composite plates. Journal of Applied Mechanics, 51:745-52, 1984. 
[27] J.N. Reddy and D.H. Robbins. Theories and computational models for composite laminates. Applied Mechanics Reviews, 47:147-165, 1994.

[28] A. Tessler, M. Di Sciuva, and M. Gherlone. A consistent refinement of first-order shear deformation theory for laminated composite and sandwich plates using improved zigzag kinematics. Journal of Mechanics of Materials and Structures, 5:341-367, 2010.

[29] A. Tessler, M. Di Sciuva, and M. Gherlone. A homogeneous limit methodology and refinements of computationally efficient zigzag theory for homogeneous, laminated composite, and sandwich plates. Numerical Methods for Partial Differential Equations, 27:208-229, 2011.

[30] A. Tessler, D.W. Sleight, and J.T. Wang. Refined zigzag theory for homogeneous, laminated composite, and sandwich beams derived from reissner's mixed variational principle. Meccanica, 50:2621-2648, 2015.

[31] A. Toledano and H. Murakami. A high-order laminated plate theory with improved in-plane responses. International Journal of Solids and Structures, 23(1):111-31, 1987.

[32] M. Touratier. An efficient standard plate theory. International Journal of Engineering Science, 29(8):901-16, 1991.

[33] D. Versino, M. Gherlone, and Di Sciuva M. Four-node s shell element for doubly curved multilayered composites based on the the refined zigzag theory. Composites Structures, 11B:392-402, 2014.

[34] D. Versino, M. Gherlone, M. Mattone, M. Di Sciuva, and A. Tessler. C0 triangular elements based on the refined zigzag theory for multilayered composite and sandwich plates. Composites Part B, Engineering, 44B:218-230, 2013.

[35] L. Vu-Quoc and X.G. Tan. Effcient hybrid-eas solid element for accurate stress prediction in thick laminated beams, plates, and shells. Computer Methods in Applied Mechanics and Engineering, 253:337-355, 2013.

[36] O.C. Zienkiewicz and R.L. Taylor. The finite element method. Vol II: Solid Mechanics. Butterworth heinemann, 2000. 


\section{Appendix}

The trigonometric shear deformation theory (TSDT) kinematic assumption for plates [32] may be recast for symmetric laminates as $(\alpha=1,2)$

$$
u_{\alpha}^{t r}(z)=-z \frac{\partial u_{z}}{\partial X_{\alpha}}+\sin \left(\frac{\pi z}{h}\right) h \varphi_{\alpha}
$$

where $u_{z}$ are the transverse displacement of the middle surface (assumed uniform across the thickness) and $\pm h \varphi_{\alpha}$ are additional in-plane displacements at the external surfaces. The associated transverse shear strains are

$$
\gamma_{\alpha}^{t r}=\frac{\partial u_{z}}{\partial X_{\alpha}}+\frac{\partial u_{\alpha}}{\partial z}=\cos \left(\frac{\pi z}{h}\right) \pi \varphi_{\alpha}
$$

where $\gamma_{\alpha}$ vanish at external surfaces.

The first order shear deformation theory (FSDT) assumes a linear variation of in-plane displacements in terms of the normal plate rotation $\left(\theta_{\alpha}\right)$ and leads to a constant shear strain

$$
\begin{aligned}
u_{\alpha}^{f}(z) & =z \theta_{\alpha} \\
\gamma_{\alpha}^{f} & =\frac{\partial u_{z}}{\partial X_{\alpha}}+\theta_{\alpha}
\end{aligned}
$$

The augmented FSDT kinematic defined by equation (48) particularized for flat plates is

$$
\begin{aligned}
u_{\alpha}^{f a}(z) & =z \theta_{\alpha}+\psi_{\alpha} \phi_{\alpha}(z)=z \theta_{\alpha}+\psi_{\alpha}\left[h \sin \left(\frac{\pi z}{h}\right)-2 z\right] \\
\gamma_{\alpha}^{f a} & =\frac{\partial u_{z}}{\partial X_{\alpha}}+\theta_{\alpha}+\psi_{\alpha} \frac{\partial \phi_{\alpha}(z)}{\partial z}=\gamma^{f}-2 \psi_{\alpha}+\pi \psi_{\alpha} \cos \left(\frac{\pi z}{h}\right)
\end{aligned}
$$

Note that both the TSDT and the augmented FSDT has a cosine variation of the shear strain across the thickness, and are identical only if $\varphi_{\alpha}=\psi_{\alpha}$ and $\gamma^{f}=2 \psi_{\alpha}$ (i.e. when the augmented FSDT shear vanishes at external surfaces). Now equaling the average transverse shear strain across the thickness for TSDT and augmented FSDT approaches

$$
\begin{aligned}
\bar{\gamma}^{t r}=\frac{1}{h} \int_{h} \gamma^{t r} d z & =\frac{1}{h} \int_{h} \gamma^{f a} d z=\bar{\gamma}^{f a} \\
\frac{1}{h} \varphi_{\alpha} \pi \int_{h} \cos \frac{\pi z}{h} d z & =\gamma^{f}+\frac{1}{h} \psi_{\alpha} \int_{h} \frac{\partial \phi_{\alpha}(z)}{\partial z} d z \\
\left.\varphi_{x} \sin \frac{\pi z}{h}\right|_{-h / 2} ^{h / 2} & =\gamma^{f}+\frac{1}{h} \psi_{\alpha}\left[\phi_{\alpha}(h / 2)-\phi_{\alpha}(-h / 2)\right] \\
2 \varphi_{x} & =\gamma_{\alpha}^{f}
\end{aligned}
$$


As expected the additional displacements for the FSDT do not modify the average shear strain (this is true for any continuous additional displacements that satisfies (41)) and both approaches have the same average shear strain as long as eq. (74) is satisfied. If we now compare the in-plane displacements at the external surfaces when (74) is satisfied

$$
\begin{aligned}
& u_{\alpha}^{t r}\left(z=\frac{h}{2}\right)=-\frac{h}{2} \frac{\partial u_{z}}{\partial X_{\alpha}}+h \varphi_{\alpha}=\frac{h}{2}\left(-\frac{\partial u_{z}}{\partial X_{\alpha}}+2 \varphi_{\alpha}\right) \\
& u_{\alpha}^{f a}\left(z=\frac{h}{2}\right)=\frac{h}{2} \theta_{\alpha}=\frac{h}{2}\left(-\frac{\partial u_{z}}{\partial X_{\alpha}}+\gamma_{\alpha}^{f}\right)=\frac{h}{2}\left(-\frac{\partial u_{z}}{\partial X_{\alpha}}+2 \varphi_{x}\right)
\end{aligned}
$$

This shows that if eq. (74) is satisfied, the proposed augmented FSDT kinematic leads to the same in-plane displacement at the plate external surfaces.

Note that for flat plates the TSDT is a three-parameter theory while the augmented FSDT leads to a five-parameter theory that does not assure that transverse shear strains will vanish at the external surfaces, and a more flexible behavior is expected. 